JOURNAL OF

FUNCTION SPACES AND APPLICATIONS

Volume 9, Number 1 (2011), 67-107 (c) 2011, Scientific Horizon http://www.jfsa.net

\title{
On boundedness and compactness of a certain class of kernel operators
}

\author{
Elena P. Ushakova \\ (Communicated by Oleg V. Besov)
}

2000 Mathematics Subject Classification. Primary 26D10; Secondary 26D15, $26 \mathrm{D} 07$.

Keywords and phrases. Integral operators; Lebesgue spaces; weights; boundedness; compactness.

\footnotetext{
Abstract. New conditions for $L_{p}[0, \infty)-L_{q}[0, \infty)$ boundedness and compactness $(1<p, q<\infty)$ of the map $f \rightarrow w(x) \int_{a(x)}^{b(x)} k(x, y) f(y) v(y) d y$ with locally integrable weight functions $v, w$ and a positive continuous kernel $k(x, y)$ from the Oinarov's class are obtained.
}

\section{Introduction}

Let $1<p<\infty$ and $\|f\|_{p}:=\left(\int_{0}^{\infty}|f(x)|^{p} d x\right)^{1 / p}$. Denote $L_{p}$ the Lebesgue space of all measurable functions on $\mathbb{R}^{+}:=[0, \infty)$ such that $\|f\|_{p}<\infty$.

Let $w(x)$ and $v(y)$ be non-negative locally integrable weight functions on $\mathbb{R}^{+}$. We study $L_{p}-L_{q}$ boundedness and compactness of an integral operator of the form

$$
\mathcal{K} f(x):=w(x) \int_{a(x)}^{b(x)} k(x, y) f(y) v(y) d y
$$


with border functions $a(x)$ and $b(x)$ satisfying the conditions

(i) $\quad a(x)$ and $b(x)$ are differentiable and strictly increasing on $(0, \infty)$;

(ii) $a(0)=b(0)=0, a(x)<b(x)$ for $0<x<\infty, a(\infty)=b(\infty)=\infty$,

and a continuous kernel $k(x, y)>0$ on $\mathcal{R}:=\{(x, y): x>0, a(x)<y<b(x)\}$ satisfying generalized Oinarov's conditions $\mathcal{O}_{b}$ or/and $\mathcal{O}_{a}$.

Definition 1.1. We write $k(x, y) \in \mathcal{O}_{b}$ if there exists a constant $D \geq 1$ independent on $x, y, z$ such that

(1.3)

$D^{-1} k(x, y) \leq k(x, b(z))+k(z, y) \leq D k(x, y), \quad z \leq x, a(x) \leq y \leq b(z)$.

Similarly, $k(x, y) \in \mathcal{O}_{a}$ if

(1.4)

$D^{-1} k(x, y) \leq k(x, a(z))+k(z, y) \leq D k(x, y), \quad x \leq z, a(z) \leq y \leq b(x)$.

Operators of the type (1.1) have been studied in [1] - [4], [6], [9] and [11]. In particular, $L_{p}-L_{q}$ boundedness and compactness criteria for $\mathcal{K}$ were obtained in [9].

Theorem 1.1. [9, Theorems 2, 3 ] Let $1<p \leq q<\infty$ and the operator $\mathcal{K}$ be defined by (1.1) with the border functions $a(x), b(x)$ satisfying (1.2). If the kernel $k(x, y) \in \mathcal{O}_{b}$, then

$$
\alpha_{1}(p, q) \mathcal{A}_{b} \leq\|\mathcal{K}\|_{L_{p} \rightarrow L_{q}} \leq \alpha_{2}(p, q) \mathcal{A}_{b},
$$

where positive constants $\alpha_{1}, \alpha_{2}$ depend only on the parameters $p, q$ and $\mathcal{A}_{b}:=\mathcal{A}_{b, 0}+\mathcal{A}_{b, 1}$ with

$$
\mathcal{A}_{b, 0}:=\sup _{t>0} \mathcal{A}_{b, 0}(t)=\sup _{t>0} \sup _{b^{-1}(a(t)) \leq s \leq t}\left(\int_{s}^{t} k^{q}(x, b(s)) w^{q}(x) d x\right)^{\frac{1}{q}}\left(\int_{a(t)}^{b(s)} v^{p^{\prime}}(y) d y\right)^{\frac{1}{p^{\prime}}},
$$

$$
\mathcal{A}_{b, 1}:=\sup _{t>0} \mathcal{A}_{b, 1}(t)=\sup _{t>0} \sup _{b^{-1}(a(t)) \leq s \leq t}\left(\int_{s}^{t} w^{q}(x) d x\right)^{\frac{1}{q}}\left(\int_{a(t)}^{b(s)} k^{p^{\prime}}(s, y) v^{p^{\prime}}(y) d y\right)^{\frac{1}{p^{\prime}}} .
$$

Moreover, $\mathcal{K}$ is compact if and only if $\mathcal{A}_{b}<\infty$ and $\lim _{s \rightarrow 0} \mathcal{A}_{b, i}(s)=$ $\lim _{s \rightarrow \infty} \mathcal{A}_{b, i}(s)=0, i=0,1$.

If the kernel $k(x, y) \in \mathcal{O}_{a}$, then

$$
\beta_{1}(p, q) \mathcal{A}_{a} \leq\|\mathcal{K}\|_{L_{p} \rightarrow L_{q}} \leq \beta_{2}(p, q) \mathcal{A}_{a}
$$


where positive constants $\beta_{1}, \beta_{2}$ depend only on the parameters $p, q$ and $\mathcal{A}_{a}:=\mathcal{A}_{a, 0}+\mathcal{A}_{a, 1}$ with

$$
\begin{aligned}
& \mathcal{A}_{a, 0}:=\sup _{s>0} \mathcal{A}_{a, 0}(s)=\sup _{s>0} \sup _{s \leq t \leq a^{-1}(b(s))}\left(\int_{s}^{t} k^{q}(x, a(t)) w^{q}(x) d x\right)^{\frac{1}{q}}\left(\int_{a(t)}^{b(s)} v^{p^{\prime}}(y) d y\right)^{\frac{1}{p^{\prime}}}, \\
& \mathcal{A}_{a, 1}:=\sup _{s>0} \mathcal{A}_{a, 1}(s)=\sup _{s>0} \sup _{s \leq t \leq a^{-1}(b(s))}\left(\int_{s}^{t} w^{q}(x) d x\right)^{\frac{1}{q}}\left(\int_{a(t)}^{b(s)} k^{p^{\prime}}(t, y) v^{p^{\prime}}(y) d y\right)^{\frac{1}{p^{\prime}}} .
\end{aligned}
$$

Besides, $\mathcal{K}$ is compact if and only if $\mathcal{A}_{a}<\infty$ and $\lim _{s \rightarrow 0} \mathcal{A}_{a, i}(s)=$ $\lim _{s \rightarrow \infty} \mathcal{A}_{a, i}(s)=0, i=0,1$.

Similar to (1.5) and (1.8) two-sided estimates for the case $1<q<p<\infty$ were obtained in [9] in a discrete form. This fact together with double supremum in the definitions of $\mathcal{A}_{a}$ and $\mathcal{A}_{b}$ may be rather inconvenient for further development.

In this work we obtain new necessary and new sufficient conditions for $L_{p}-L_{q}$ boundedness and compactness of $\mathcal{K}$ (see Sections 3 and 4) which become a criterion either under some additional requirements on weight functions or when a kernel satisfies (1.3) and (1.4) simultaneously (see Section 5). Unlike to the above results the new criteria have one supremum if $1<p \leq q<\infty$ and a continuous form for $1<q<p<\infty$. We start the paper with Preliminaries (Section 2) and conclude by Examples (Section 6).

Throughout the paper products of the form $0 \cdot \infty$ are taken to be equal to 0 . Relations $A \ll B$ mean $A \leq c B$ with some constants $c$ depending only on parameters of summations and, possibly, on the constants of equivalence in the inequalities of the type (1.3). We write $A \approx B$ instead of $A \ll B \ll A$ or $A=c B$. $\mathbb{Z}$ denotes the set of all integers and $\chi_{E}$ stands for a characteristic function (indicator) of a subset $E \subset \mathbb{R}^{+}$. Also we make use of marks : $=$ and $=$ : for introducing new quantities and suppose $p^{\prime}:=p /(p-1)$ for $1<p<\infty$ and $r:=p q /(p-q)$ for $1<q<p<\infty$.

\section{Preliminaries}

Here we collect some statements, which are necessary to prove our results. We start with Lemma 2.1 on a block-diagonal operator.

Lemma 2.1 ([10, Lemma 1]). Let $0<q<p<\infty$, let $U=\bigsqcup_{k} U_{k}$ and $V=\bigsqcup_{k} V_{k}$ be unions of non-overlapping measurable sets and let $T=\sum_{k} T_{k}$, where $T_{k}: L_{p}\left(U_{k}\right) \rightarrow L_{q}\left(V_{k}\right)$. Then 


$$
\|T\|_{L_{p}(U) \rightarrow L_{q}(V)} \approx\left(\sum_{k}\left\|T_{k}\right\|_{L_{p}\left(U_{k}\right) \rightarrow L_{q}\left(V_{k}\right)}^{r}\right)^{1 / r}, \quad 1 / r=1 / q-1 / p .
$$

The next Theorem 2.1 is a known result for the Hardy type operator

$$
K f(x):=w(x) \int_{c}^{x} k(x, y) f(y) v(y) d y, \quad 0 \leq c \leq x \leq d \leq \infty,
$$

with the kernel $k(x, y) \geq 0$ satisfying the Oinarov's condition of the form: there exists a constant $D \geq 1$ independent on $x, y, z$ such that

$$
D^{-1} k(x, y) \leq k(x, z)+k(z, y) \leq D k(x, y), \quad 0 \leq c \leq y \leq z \leq x \leq d \leq \infty .
$$

Theorem 2.1. ([8, Theorem 2]) Let $1<q<p<\infty$. Then $\|K\|_{L_{p}(c, d) \rightarrow L_{q}(c, d)} \approx B_{0}+B_{1}$, where

$$
\begin{aligned}
& B_{0}:=\left(\int_{c}^{d}\left[\int_{t}^{d} k^{q}(x, t) w^{q}(x) d x\right]^{\frac{r}{q}}\left[\int_{c}^{t} v^{p^{\prime}}(y) d y\right]^{\frac{r}{q^{\prime}}} v^{p^{\prime}}(t) d t\right)^{\frac{1}{r}}, \\
& B_{1}:=\left(\int_{c}^{d}\left[\int_{t}^{d} w^{q}(x) d x\right]^{\frac{r}{p}}\left[\int_{c}^{t} k^{p^{\prime}}(t, y) v^{p^{\prime}}(y) d y\right]^{\frac{r}{p^{\prime}}} w^{q}(t) d t\right)^{\frac{1}{r}} .
\end{aligned}
$$

The result of Theorem 2.1 can be extended to a more general then (2.1) operator $K_{b}: L_{p}(b(c), b(d)) \rightarrow L_{q}(c, d)$ of the form

$$
K_{b} f(x):=w(x) \int_{b(c)}^{b(x)} k(x, y) f(y) v(y) d y, \quad 0 \leq c \leq x \leq d \leq \infty,
$$

with a differentiable and strictly increasing on $[c, d)$ function $b(x) \geq 0$ and a non-negative kernel $k(x, y)$ satisfying the condition: there exists a constant $D \geq 1$ such that

$D^{-1} k(x, y) \leq k(x, b(z))+k(z, y) \leq D k(x, y), \quad\left\{\begin{array}{l}0 \leq c \leq z \leq x \leq d \leq \infty \\ 0 \leq b(c) \leq y \leq b(z)\end{array}\right.$

Corollary 2.1. Let $1<q<p<\infty, r=p q /(p-q)$ and the operator $K_{b}$ be given by (2.3) with a differentiable and strictly increasing on $[c, d)$ function $b(x) \geq 0$ and a non-negative kernel $k(x, y) \in(2.4)$. Then

$$
\left\|K_{b}\right\|_{L_{p}(b(c), b(d)) \rightarrow L_{q}(c, d)} \approx B_{b, 0}+B_{b, 1},
$$


where

$$
B_{b, 0}:=\left(\int_{b(c)}^{b(d)}\left[\int_{b^{-1}(t)}^{d} k^{q}(x, t) w^{q}(x) d x\right]^{\frac{r}{q}}\left[\int_{b(c)}^{t} v^{p^{\prime}}(y) d y\right]^{\frac{r}{q^{\prime}}} v^{p^{\prime}}(t) d t\right)^{\frac{1}{r}},
$$

$$
B_{b, 1}:=\left(\int_{c}^{d}\left[\int_{t}^{d} w^{q}(x) d x\right]^{\frac{r}{p}}\left[\int_{b(c)}^{b(t)} k^{p^{\prime}}(t, y) v^{p^{\prime}}(y) d y\right]^{\frac{r}{p^{\prime}}} w^{q}(t) d t\right)^{\frac{1}{r}} .
$$

By duality and Theorem 2.1 we can obtain norm estimates for the operator

$$
K_{a} f(x):=w(x) \int_{a(x)}^{a(d)} k(x, y) f(y) v(y) d y, \quad 0 \leq c \leq x \leq d \leq \infty,
$$

with a differentiable and strictly increasing on $[c, d)$ function $a(x) \geq 0$ and a non-negative kernel $k(x, y)$ satisfying the condition: there exists a constant $D \geq 1$ such that

$D^{-1} k(x, y) \leq k(x, a(z))+k(z, y) \leq D k(x, y), \quad\left\{\begin{array}{l}0 \leq c \leq x \leq z \leq d \leq \infty \\ 0 \leq a(z) \leq y \leq a(d) .\end{array}\right.$

Corollary 2.2. Let $1<q<p<\infty, r=p q /(p-q)$ and the operator $K_{a}$ be defined by (2.8) with a differentiable and strictly increasing on $[c, d)$ function $a(x) \geq 0$ and a non-negative kernel $k(x, y) \in(2.9)$. Then $\left\|K_{a}\right\|_{L_{p}(a(c), a(d)) \rightarrow L_{q}(c, d)} \approx B_{a, 0}+B_{a, 1}$, where

$$
\begin{aligned}
B_{a, 0} & :=\left(\int_{a(c)}^{a(d)}\left[\int_{c}^{a^{-1}(t)} k^{q}(x, t) w^{q}(x) d x\right]^{\frac{r}{q}}\left[\int_{t}^{a(d)} v^{p^{\prime}}(y) d y\right]^{\frac{r}{q^{\prime}}} v^{p^{\prime}}(t) d t\right)^{\frac{1}{r}}, \\
B_{a, 1} & :=\left(\int_{c}^{d}\left[\int_{c}^{t} w^{q}(x) d x\right]^{\frac{r}{p}}\left[\int_{a(t)}^{a(d)} k^{p^{\prime}}(t, y) v^{p^{\prime}}(y) d y\right]^{\frac{r}{p^{\prime}}} w^{q}(t) d t\right)^{\frac{1}{r}} .
\end{aligned}
$$

Now we can state the next auxiliary result of the paper.

Lemma 2.2. Let $1<q<p<\infty$. Suppose $b(x) \geq 0$ is differentiable and strictly increasing on $[c, d)$, and a satisfying (2.4) non-negative function $k(x, y)$ is continuous on $\mathcal{R}_{b}:=\{(x, y): x>0, b(c)<y<b(x)\}$. Then we 
have

$$
\begin{aligned}
& B_{b, 0} \ll \mathcal{B}_{b, 0}:=\left(\int_{b(c)}^{b(d)}\left[\int_{t}^{b(d)}\left\{\int_{b^{-1}(y)}^{d} k^{q}(x, y) w^{q}(x) d x\right\}^{p^{\prime}} v^{p^{\prime}}(y) d y\right]^{\frac{r}{q^{\prime}}} \times\right. \\
& \times {\left.\left[\int_{b^{-1}(t)}^{d} k^{q}(x, t) w^{q}(x) d x\right]^{p^{\prime}-\frac{r}{q^{\prime}}} v^{p^{\prime}}(t) d t\right)^{\frac{1}{r}}, } \\
& B_{b, 1} \ll \mathbb{B}_{b, 1}:=\left(\int_{c}^{d}\left[\int_{c}^{t}\left\{\int_{b(c)}^{b(x)} k^{p^{\prime}}(x, y) v^{p^{\prime}}(y) d y\right\}^{q} w^{q}(x) d x\right]^{\frac{r}{p}} \times\right. \\
&\left.\times\left[\int_{b(c)}^{b(t)} k^{p^{\prime}}(t, y) v^{p^{\prime}}(y) d y\right]^{q-\frac{r}{p}} w^{q}(t) d t\right)^{\frac{1}{r}},
\end{aligned}
$$

where $B_{b, 0}$ and $B_{b, 1}$ are defined by (2.6) and (2.7) respectively.

For the proof of Lemma 2.2, we refer first to the paper [5].

Definition 2.1. ([5, Definition 2.2(a)]) A nonnegative sequence $\left\{a_{k}\right\}_{k \in \mathbb{Z}}$ is said to be strongly increasing (strongly decreasing), denoted by $a_{k} \uparrow \uparrow$ $\left(a_{k} \downarrow\right)$, if

$$
\inf _{k \in \mathbb{Z}} \frac{a_{k+1}}{a_{k}}>1 \quad\left(\sup _{k \in \mathbb{Z}} \frac{a_{k+1}}{a_{k}}<1\right) .
$$

Proposition 2.1. ([5, Proposition 2.1]) Let $\left\{a_{k}\right\}_{k \in \mathbb{Z}},\left\{\sigma_{k}\right\}_{k \in \mathbb{Z}}$ and $\left\{\tau_{k}\right\}_{k \in \mathbb{Z}}$ be nonnegative sequences and $0<p<\infty$.

(a) If $\sigma_{k} \uparrow$, then $\left(\sum_{k \in \mathbb{Z}}\left[\sum_{m \geq k} a_{m}\right]^{p} \sigma_{k}^{p}\right)^{\frac{1}{p}} \ll\left(\sum_{m \in \mathbb{Z}}\left[a_{m} \sigma_{m}\right]^{p}\right)^{\frac{1}{p}}$.

(b) If $\tau_{k} \downarrow$, then $\left(\sum_{k \in \mathbb{Z}}\left[\sum_{m \leq k} a_{m}\right]^{p} \tau_{k}^{p}\right)^{\frac{1}{p}} \ll\left(\sum_{m \in \mathbb{Z}}\left[a_{m} \tau_{m}\right]^{p}\right)^{\frac{1}{p}}$.

Proof. [Proof of Lemma 2.2.] By the substitution $\tau=b^{-1}(t)$ we reduce $B_{b, 0}, B_{b, 1}$ to the constants $B_{0}$ and $B_{1}$ respectively. Analogously we arrive to $\mathcal{B}_{0}:=\mathcal{B}_{b(x)=x, 0}$ and $\mathbb{B}_{1}:=\mathbb{B}_{b(x)=x, 1}$. Now it is sufficient to prove (2.10), (2.11) for the case $b(x)=x$ only. Note that under this condition the kernel $k(x, t)=k(x, b(\tau))=: k_{b}(x, \tau)$ becomes satisfying (2.2). Moreover, without loss of generality we can assume that $k_{b}(x, \tau)$ is non-decreasing with respect to the variable $x$ and non-increasing with respect to $y$. Otherwise we can consider the kernel $\bar{k}_{b}(x, \tau)=\sup _{\tau \leq s \leq x} \widetilde{k}_{b}(s, \tau)$, where $\widetilde{k}_{b}(s, \tau)=$ $\sup _{\tau \leq t \leq s} k_{b}(s, t)$, which satisfies the pointed monotonicity properties and $k_{b}(x, \tau) \leq \bar{k}_{b}(x, \tau) \leq D^{2} k_{b}(x, \tau)$ (for details see [7, Lemma 3]). 
For the proof of (2.10) we put

$$
t_{k}=\inf \left\{t: \int_{t}^{d} k^{q}(x, t) w^{q}(x) d x \leq 2^{-k}\right\}, \quad k \in \mathbb{Z},
$$

and denote $\mathbb{Z}_{1}:=\left\{k \in \mathbb{Z}: t_{k}<t_{k+1}\right\}$. Observe that the function $W_{q}(t):=$ $\int_{t}^{d} k^{q}(x, t) w^{q}(x) d x$ is continuous, non-increasing and such that

$$
2^{-k-1} \leq W_{q}(t) \leq 2^{-k}, \quad t_{k} \leq t \leq t_{k+1} .
$$

Put $V(t):=\int_{c}^{t} v^{p^{\prime}}(y) d y$. We have

$$
\begin{aligned}
B_{0}^{r} & =\frac{p^{\prime}}{r} \sum_{k \in \mathbb{Z}_{1}} \int_{t_{k}}^{t_{k+1}}\left[W_{q}(t)\right]^{\frac{r}{q}} d[V(t)]^{\frac{r}{p^{\prime}}} \ll \sum_{k \in \mathbb{Z}_{1}} 2^{-k \frac{r}{q}} \int_{t_{k}}^{t_{k+1}} d[V(t)]^{\frac{r}{p^{\prime}}} \\
& \leq \sum_{k \in \mathbb{Z}_{1}} 2^{-k \frac{r}{q}}\left[V\left(t_{k+1}\right)\right]^{\frac{r}{p^{\prime}}}=\sum_{k \in \mathbb{Z}_{1}} 2^{-k \frac{r}{q}}\left(\sum_{m \leq k} \int_{t_{m}}^{t_{m+1}} v^{p^{\prime}}(y) d y\right)^{\frac{r}{p^{\prime}}} .
\end{aligned}
$$

Applying Proposition 2.1(b) we find that

$$
\begin{aligned}
B_{0}^{r} & \ll \sum_{k \in \mathbb{Z}_{1}} 2^{-k \frac{r}{q}}\left(\int_{t_{k}}^{t_{k+1}} v^{p^{\prime}}(y) d y\right)^{\frac{r}{p^{\prime}}} \\
& \stackrel{(2.12)}{\simeq} \sum_{k \in \mathbb{Z}_{1}} 2^{k \frac{r}{q^{\prime}}}\left(\int_{t_{k}}^{t_{k+1}} v^{p^{\prime}}(y) d y\right)^{\frac{r}{p^{\prime}}}\left[W_{q}\left(t_{k+1}\right)\right]^{r} \\
& \leq \sum_{k \in \mathbb{Z}_{1}} 2^{k \frac{r}{q^{\prime}}}\left(\int_{t_{k}}^{t_{k+1}}\left[W_{q}(y)\right]^{p^{\prime}} v^{p^{\prime}}(y) d y\right)^{\frac{r}{p^{\prime}}} \\
& \approx \sum_{k \in \mathbb{Z}_{1}}\left[W_{q}\left(t_{k}\right)\right]^{-\frac{r}{q^{\prime}}} \int_{t_{k}}^{t_{k+1}}\left(\int_{t}^{t_{k+1}}\left[W_{q}(y)\right]^{p^{\prime}} v^{p^{\prime}}(y) d y\right)^{\frac{r}{q^{\prime}}}\left[W_{q}(t)\right]^{p^{\prime}} v^{p^{\prime}}(t) d t \\
& \leq \sum_{k \in \mathbb{Z}_{1}} \int_{t_{k}}^{t_{k+1}}\left(\int_{t}^{d}\left[W_{q}(y)\right]^{p^{\prime}} v^{p^{\prime}}(y) d y\right)^{\frac{r}{q^{\prime}}}\left[W_{q}(t)\right]^{p^{\prime}-\frac{r}{q^{\prime}}} v^{p^{\prime}}(t) d t=\mathcal{B}_{0}^{r} .
\end{aligned}
$$

Analogously, to prove (2.11) we put

$$
t_{l}=\sup \left\{t: \int_{c}^{t} k^{p^{\prime}}(t, y) v^{p^{\prime}}(y) d y \leq 2^{l}\right\}, \quad l \in \mathbb{Z},
$$

and $\mathbb{Z}_{2}:=\left\{l \in \mathbb{Z}: t_{l}<t_{l+1}\right\}$. Note that $V_{p^{\prime}}(t):=\int_{c}^{t} k^{p^{\prime}}(t, y) v^{p^{\prime}}(y) d y$ is continuous, non-decreasing and such that 


$$
2^{l} \leq V_{p^{\prime}}(t) \leq 2^{l+1}, \quad t_{l} \leq t \leq t_{l+1} .
$$

Put $W(t):=\int_{t}^{d} w^{q}(x) d x$. Then

$$
\begin{array}{r}
B_{1}^{r}=\frac{q}{r} \sum_{l \in \mathbb{Z}_{2}} \int_{t_{l}}^{t_{l+1}}\left[V_{p^{\prime}}(t)\right]^{\frac{r}{p^{\prime}}} d\left(-[W(t)]^{\frac{r}{q}}\right) \ll \sum_{l \in \mathbb{Z}_{2}} 2^{l \frac{r}{p^{\prime}}} \int_{t_{l}}^{t_{l+1}} d\left(-[W(t)]^{\frac{r}{q}}\right) \\
\leq \sum_{l \in \mathbb{Z}_{2}} 2^{l \frac{r}{p^{\prime}}}\left[W\left(t_{l}\right)\right]^{\frac{r}{q}}=\sum_{k \in \mathbb{Z}_{2}} 2^{l \frac{r}{p^{\prime}}}\left(\sum_{m \geq l} \int_{t_{m}}^{t_{m+1}} w^{q}(x) d x\right)^{\frac{r}{q}} .
\end{array}
$$

Applying Proposition 2.1(a) we obtain

$$
\begin{aligned}
B_{1}^{r} & \ll \sum_{l \in \mathbb{Z}_{2}} 2^{l \frac{r}{p^{\prime}}}\left(\int_{t_{l}}^{t_{l+1}} w^{q}(x) d x\right)^{\frac{r}{q}} \stackrel{(2.13)}{\simeq} \sum_{l \in \mathbb{Z}_{2}} 2^{-l \frac{r}{p}}\left(\int_{t_{l}}^{t_{l+1}} w^{q}(x) d x\right)^{\frac{r}{q}}\left[V_{p^{\prime}}\left(t_{l}\right)\right]^{r} \\
& \leq \sum_{l \in \mathbb{Z}_{2}} 2^{-l \frac{r}{p}}\left(\int_{t_{l}}^{t_{l+1}}\left[V_{p^{\prime}}(x)\right]^{q} w^{q}(x) d x\right)^{\frac{r}{q}} \\
& \approx \sum_{l \in \mathbb{Z}_{2}} V_{p^{\prime}}\left(t_{l+1}\right)^{-\frac{r}{p}} \int_{t_{l}}^{t_{l+1}}\left(\int_{t_{l}}^{t}\left[V_{p^{\prime}}(x)\right]^{q} w^{q}(x) d x\right)^{\frac{r}{p}}\left[V_{p^{\prime}}(t)\right]^{q} w^{q}(t) d t \\
& \leq \sum_{l \in \mathbb{Z}_{2}} \int_{t_{l}}^{t_{l+1}}\left(\int_{c}^{t}\left[V_{p^{\prime}}(x)\right]^{q} w^{q}(x) d x\right)^{\frac{r}{p}}\left[V_{p^{\prime}}(t)\right]^{q-\frac{r}{p}} w^{q}(t) d t=\mathbb{B}_{1}^{r} .
\end{aligned}
$$

Analogous result is true for the constants $B_{a, 0}$ and $B_{a, 1}$.

Lemma 2.3. Let $1<q<p<\infty$. Suppose that a function $a(x) \geq 0$ is differentiable and strictly increasing on $[c, d)$, and a continuous function $k(x, y) \geq 0$ on $\mathcal{R}_{a}:=\{(x, y): x>0, a(x)<y<a(d)\}$ is from the class (2.9). Then

$$
\begin{aligned}
B_{a, 0} \ll \mathcal{B}_{a, 0}:= & \left(\int_{a(c)}^{a(d)}\left[\int_{a(c)}^{t}\left\{\int_{c}^{a^{-1}(y)} k^{q}(x, y) w^{q}(x) d x\right\}^{p^{\prime}} v^{p^{\prime}}(y) d y\right]^{\frac{r}{q^{\prime}}} \times\right. \\
& \left.\times\left[\int_{c}^{a^{-1}(t)} k^{q}(x, t) w^{q}(x) d x\right]^{p^{\prime}-\frac{r}{q^{\prime}}} v^{p^{\prime}}(t) d t\right)^{\frac{1}{r}},
\end{aligned}
$$




$$
\begin{aligned}
B_{a, 1} \ll \mathbb{B}_{a, 1}:= & \left(\int_{c}^{d}\left[\int_{t}^{d}\left\{\int_{a(x)}^{a(d)} k^{p^{\prime}}(x, y) v^{p^{\prime}}(y) d y\right\} w^{q}(x) d x\right]^{\frac{r}{p}} \times\right. \\
& \left.\left.\times\left[\int_{a(t)}^{a(d)} k^{p^{\prime}}(t, y) v^{p^{\prime}}(y) d y\right]^{q-\frac{r}{p}} w^{(} t\right) d t\right)^{\frac{1}{r}},
\end{aligned}
$$

where $B_{a, 0}, B_{a, 1}$ are defined by (2.10) and (2.10) respectively.

We conclude this section by the following two statements.

Lemma 2.4. [11, Lemma 1] Let $0<q<p<\infty, p>1,1 / r=1 / q-1 / p$ and $0 \leq c<d \leq \infty, 0 \leq a<\infty$. Suppose that the function $b(x)$ is differentiable, strictly increasing and such that $a \leq b(x)<\infty, x \in[c, d)$. Let the operator $S$ be defined by $S f(x):=w(x) \int_{a}^{b(x)} f(y) v(y) d y$. Then

$$
\|S\|_{L_{p}(a, b(d)) \rightarrow L_{q}(c, d)}^{r} \approx \int_{c}^{d}\left(\int_{t}^{d} w^{q}(x) d x\right)^{\frac{r}{p}}\left(\int_{a}^{b(t)} v^{p^{\prime}}(y) d y\right)^{\frac{r}{p^{\prime}}} w^{q}(t) d t,
$$

$$
\approx \int_{c}^{d}\left(\int_{c}^{t}\left[\int_{a}^{b(x)} v^{p^{\prime}}(y) d y\right]^{q} w^{q}(x) d x\right)^{\frac{r}{p}}\left(\int_{a}^{b(t)} v^{p^{\prime}}(y) d y\right)^{q-\frac{r}{p}} w^{q}(t) d t .
$$

Lemma 2.5. [11, Lemma 2] Let $0<q<p<\infty, p>1,1 / r=1 / q-1 / p$ and $0 \leq c<d \leq \infty, 0<b \leq \infty$. Suppose that the function $a(x)$ is differentiable, strictly increasing and such that $0<a(x) \leq b, x \in(c, d]$. Let the operator $T$ be defined by $T f(x):=w(x) \int_{a(x)}^{b} f(y) v(y) d y$. Then

$$
\|T\|_{L_{p}(a(c), b) \rightarrow L_{q}(c, d)}^{r} \approx \int_{c}^{d}\left(\int_{c}^{t} w^{q}(x) d x\right)^{\frac{r}{p}}\left(\int_{a(t)}^{b} v^{p^{\prime}}(y) d y\right)^{\frac{r}{p^{\prime}}} w^{q}(t) d t,
$$

$$
\approx \int_{c}^{d}\left(\int_{t}^{d}\left[\int_{a(x)}^{b} v^{p^{\prime}}(y) d y\right]^{q} w^{q}(x) d x\right)^{\frac{r}{p}}\left(\int_{a(t)}^{b} v^{p^{\prime}}(y) d y\right)^{q-\frac{r}{p}} w^{q}(t) d t .
$$




\section{The main result}

Let functions $\sigma(x)$ and $\rho(y)$ on $\mathbb{R}^{+} \cup\{+\infty\}$ be such that $a(x) \leq \sigma(x) \leq$ $b(x), b^{-1}(y) \leq \rho(y) \leq a^{-1}(y)$ and be fairway-functions satisfying the following

Definition 3.1. Given boundary functions $a(x)$ and $b(x)$ satisfying the conditions (1.2), numbers $p, q \in(1, \infty)$, a continuous kernel $0<$ $k(x, y)<\infty$ a.e on $\mathcal{R}=\{(x, y): x>0, a(x)<y<b(x)\}$ and weight functions $0<v, w<\infty$ a.e. on $\mathbb{R}^{+}$such that for any fixed $x>0$ the function $k^{p^{\prime}}(x, y) v^{p^{\prime}}(y)$ is locally integrable on $\mathbb{R}^{+}$with respect to the variable $y$ as well as for any $y>0$ the function $k^{q}(x, y) w^{q}(x)$ is locally integrable on $\mathbb{R}^{+}$with respect to $x$, we define two fairways - the functions $\sigma(x)$ and $\rho(y)$ such that $a(x)<\sigma(x)<b(x), b^{-1}(y)<\rho(y)<a^{-1}(y)$ and

$$
\begin{gathered}
\int_{a(x)}^{\sigma(x)} k^{p^{\prime}}(x, y) v^{p^{\prime}}(y) d y=\int_{\sigma(x)}^{b(x)} k^{p^{\prime}}(x, y) v^{p^{\prime}}(y) d y \quad \text { for all } x>0 \\
\int_{b^{-1}(y)}^{\rho(y)} k^{q}(x, y) w^{q}(x) d x=\int_{\rho(y)}^{a^{-1}(y)} k^{q}(x, y) w^{q}(x) d x \quad \text { for all } y>0 .
\end{gathered}
$$

By assumptions of the definition the fairways $\sigma(x)$ and $\rho(y)$ are continuous functions. Put

$$
\begin{gathered}
\Theta(t):=\Theta^{-}(t) \cup \Theta^{+}(t), \quad \Theta^{-}(t):=\left[b^{-1}(t), \rho(t)\right), \quad \Theta^{+}(t):=\left[\rho(t), a^{-1}(t)\right), \\
\vartheta(t):=\vartheta^{-}(t) \cup \vartheta^{+}(t), \quad \vartheta^{-}(t):=[a(\rho(t)), t), \quad \vartheta^{+}(t):=[t, b(\rho(t))), \\
\delta(t):=\delta^{-}(t) \cup \delta^{+}(t), \quad \delta^{-}(t):=\left[b^{-1}(\sigma(t)), t\right), \quad \delta^{+}(t):=\left[t, a^{-1}(\sigma(t))\right), \\
\Delta(t):=\Delta^{-}(t) \cup \Delta^{+}(t), \quad \Delta^{-}(t):=[a(t), \sigma(t)), \quad \Delta^{+}(t):=[\sigma(t), b(t))
\end{gathered}
$$

and denote

$$
\begin{aligned}
& \mathcal{A}_{\rho}^{ \pm}:=\sup _{t>0} \mathcal{A}_{\rho}^{ \pm}(t)=\sup _{t>0}\left(\int_{\Theta(t)} k^{q}(x, t) w^{q}(x) d x\right)^{\frac{1}{q}}\left(\int_{\vartheta^{ \pm}(t)} v^{p^{\prime}}(y) d y\right)^{\frac{1}{p^{\prime}}}, \\
& \mathcal{A}_{\sigma}^{ \pm}:=\sup _{t>0} \mathcal{A}_{\sigma}^{ \pm}(t)=\sup _{t>0}\left(\int_{\delta^{ \pm}(t)} w^{q}(x) d x\right)^{\frac{1}{q}}\left(\int_{\Delta(t)} k^{p^{\prime}}(t, y) v^{p^{\prime}}(y) d y\right)^{\frac{1}{p^{\prime}}},
\end{aligned}
$$




$$
\begin{aligned}
& \mathcal{B}_{\rho}^{ \pm}:=\left(\int_{0}^{\infty} \mathcal{B}_{\rho}^{ \pm}(t) d t\right)^{\frac{1}{r}}=\left(\int_{0}^{\infty}\left[\int_{\Theta(t)} k^{q}(x, t) w^{q}(x) d x\right]^{\frac{r}{q}} \times\right. \\
& \left.\times\left[\int_{\vartheta^{ \pm}(t)} v^{p^{\prime}}(y) d y\right]^{\frac{r}{q^{\prime}}} v^{p^{\prime}}(t) d t\right)^{\frac{1}{r}} \\
& \mathcal{B}_{\sigma}^{ \pm}:=\left(\int_{0}^{\infty} \mathcal{B}_{\sigma}^{ \pm}(t) d t\right)^{\frac{1}{r}}=\left(\int_{0}^{\infty}\left[\int_{\delta^{ \pm}(t)} w^{q}(x) d x\right]^{\frac{r}{p}} \times\right. \\
& \left.\times\left[\int_{\Delta(t)} k^{p^{\prime}}(t, y) v^{p^{\prime}}(y) d y\right]^{\frac{r}{p^{\prime}}} w^{q}(t) d t\right)^{\frac{1}{r}} \\
& \mathcal{A}_{\rho}:=\sup _{t>0} \mathcal{A}_{\rho}(t)=\sup _{t>0}\left(\int_{\Theta(t)} k^{q}(x, t) w^{q}(x) d x\right)^{\frac{1}{q}}\left(\int_{\vartheta(t)} v^{p^{\prime}}(y) d y\right)^{\frac{1}{p^{\prime}}}, \\
& \mathcal{A}_{\sigma}:=\sup _{t>0} \mathcal{A}_{\sigma}(t)=\sup _{t>0}\left(\int_{\delta(t)} w^{q}(x) d x\right)^{\frac{1}{q}}\left(\int_{\Delta(t)} k^{p^{\prime}}(t, y) v^{p^{\prime}}(y) d y\right)^{\frac{1}{p^{\prime}}}, \\
& \mathcal{B}_{\rho}:=\left(\int_{0}^{\infty} \mathcal{B}_{\rho}(t) d t\right)^{\frac{1}{r}}=\left(\int_{0}^{\infty}\left[\int_{\Theta(t)} k^{q}(x, t) w^{q}(x) d x\right]^{\frac{r}{q}} \times\right. \\
& \left.\times\left[\int_{\vartheta(t)} v^{p^{\prime}}(y) d y\right]^{\frac{r}{q^{\prime}}} v^{p^{\prime}}(t) d t\right)^{\frac{1}{r}}, \\
& \mathcal{B}_{\sigma}:=\left(\int_{0}^{\infty} \mathcal{B}_{\sigma}(t) d t\right)^{\frac{1}{r}}=\left(\int_{0}^{\infty}\left[\int_{\delta(t)} w^{q}(x) d x\right]^{\frac{r}{p}} \times\right. \\
& \left.\times\left[\int_{\Delta(t)} k^{p^{\prime}}(t, y) v^{p^{\prime}}(y) d y\right]^{\frac{r}{p^{\prime}}} w^{q}(t) d t\right)^{\frac{1}{r}},
\end{aligned}
$$

where $r=p q /(p-q)$. The main result of the paper is proved in Section 4 and reads

Theorem 3.1. Let the operator $\mathcal{K}$ be defined by (1.1) with the border functions $a(x), b(x)$ satisfying (1.2) and a continuous positive kernel $k(x, y)$ on $\mathcal{R}$ from the Oinarov's type class $\mathcal{O}_{b}$. Suppose the functions $\rho(y), \sigma(x)$ on $\mathbb{R}^{+}$are strictly increasing fairways from Definition 3.1. 
(a) If $1<p \leq q<\infty$, then

$$
\alpha_{3}(p, q)\left[\mathcal{A}_{\rho}^{-}+\mathcal{A}_{\sigma}^{+}\right] \leq\|\mathcal{K}\|_{L_{p} \rightarrow L_{q}} \leq \alpha_{4}(p, q)\left[\mathcal{A}_{\rho}+\mathcal{A}_{\sigma}\right] .
$$

Moreover, if $\mathcal{K}$ is compact then $\mathcal{A}_{\rho}^{-}, \mathcal{A}_{\sigma}^{+}<\infty, \lim _{t \rightarrow 0} \mathcal{A}_{\rho}^{-}(t)=$ $\lim _{\substack{t \rightarrow 0 \\ t \rightarrow \infty}} \mathcal{A}_{\sigma}^{+}(t)=0$ and $\mathcal{K}$ is compact if $\mathcal{A}_{\rho}, \mathcal{A}_{\sigma}<\infty, \lim _{t \rightarrow \infty}<\mathcal{A}_{\rho}(t)=$ $\lim _{t \rightarrow 0} \mathcal{A}_{\sigma}(t)=0$.

(b) If $1<q<p<\infty$, then

$$
\beta_{3}(p, q)\left[\mathcal{B}_{\rho}^{-}+\mathcal{B}_{\sigma}^{+}\right] \leq\|\mathcal{K}\|_{L_{p} \rightarrow L_{q}} \leq \beta_{4}(p, q)\left[\mathcal{B}_{\rho}+\mathcal{B}_{\sigma}\right] .
$$

Moreover, the operator $\mathcal{K}$ is compact if $\mathcal{B}_{\rho}, \mathcal{B}_{\sigma}<\infty$ and if $\mathcal{K}$ is compact then $\mathcal{B}_{\rho}^{-}, \mathcal{B}_{\sigma}^{+}<\infty$.

Analogously we obtain a similar result for $\mathcal{K}$ with $k(x, y)$ satisfying the condition (1.4):

Theorem 3.2. Let the operator $\mathcal{K}$ be defined by (1.1) with the border functions $a(x), b(x)$ satisfying (1.2) and a continuous kernel $k(x, y)>0$ on $\mathcal{R}$ from the Oinarov's type class $\mathcal{O}_{a}$. Suppose that the functions $\rho(y)$, $\sigma(x)$ on $\mathbb{R}^{+}$are strictly increasing fairways from Definition 3.1.

(a) If $1<p \leq q<\infty$, then

$$
\alpha_{5}(p, q)\left[\mathcal{A}_{\rho}^{+}+\mathcal{A}_{\sigma}^{-}\right] \leq\|\mathcal{K}\|_{L_{p} \rightarrow L_{q}} \leq \alpha_{6}(p, q)\left[\mathcal{A}_{\rho}+\mathcal{A}_{\sigma}\right] .
$$

Moreover, if $\mathcal{K}$ is compact then $\mathcal{A}_{\rho}^{+}, \mathcal{A}_{\sigma}^{-}<\infty, \lim _{t \rightarrow 0} \mathcal{A}_{\rho}^{+}(t)=$ $\lim _{\substack{t \rightarrow 0 \\ t \rightarrow \infty}} \mathcal{A}_{\sigma}^{-}(t)=0$ and $\mathcal{K}$ is compact if $\mathcal{A}_{\rho}, \mathcal{A}_{\sigma}<\infty, \lim _{\substack{t \rightarrow \infty \\ t \rightarrow \infty}} \mathcal{A}_{\rho}(t)=$ $\lim _{t \rightarrow 0} \mathcal{A}_{\sigma}(t)=0$.

(b) If $1<q<p<\infty$, then

$$
\beta_{5}(p, q)\left[\mathcal{B}_{\rho}^{+}+\mathcal{B}_{\sigma}^{-}\right] \leq\|\mathcal{K}\|_{L_{p} \rightarrow L_{q}} \leq \beta_{6}(p, q)\left[\mathcal{B}_{\rho}+\mathcal{B}_{\sigma}\right] .
$$

Moreover, the operator $\mathcal{K}$ is compact if $\mathcal{B}_{\rho}, \mathcal{B}_{\sigma}<\infty$ and if $\mathcal{K}$ is compact then $\mathcal{B}_{\rho}^{+}, \mathcal{B}_{\sigma}^{-}<\infty$.

\section{Proof of Theorem 3.1}

(a) The lower estimate. Let $1<p \leq q<\infty$. It follows from (1.5) of Theorem 1.1 that

$$
\|\mathcal{K}\|_{L_{p} \rightarrow L_{q}} \approx \sup _{t>0} \sup _{b^{-1}(a(t)) \leq s \leq t}\left[\mathcal{A}_{0}(s, t)+\mathcal{A}_{1}(s, t)\right],
$$


where

$$
\begin{aligned}
& \mathcal{A}_{0}(s, t):=\left(\int_{s}^{t} k^{q}(x, b(s)) w^{q}(x) d x\right)^{\frac{1}{q}}\left(\int_{a(t)}^{b(s)} v^{p^{\prime}}(y) d y\right)^{\frac{1}{p^{\prime}}}, \\
& \mathcal{A}_{1}(s, t):=\left(\int_{s}^{t} w^{q}(x) d x\right)^{\frac{1}{q}}\left(\int_{a(t)}^{b(s)} k^{p^{\prime}}(s, y) v^{p^{\prime}}(y) d y\right)^{\frac{1}{p^{\prime}}} .
\end{aligned}
$$

Using (3.2) we find that

$$
\begin{aligned}
\mathcal{A}_{\rho}^{-}(t) & =2^{1 / q}\left(\int_{\Theta^{-}(t)} k^{q}(x, t) w^{q}(x) d x\right)^{\frac{1}{q}}\left(\int_{\vartheta^{-}(t)} v^{p^{\prime}}(y) d y\right)^{\frac{1}{p^{\prime}}} \\
& =2^{1 / q}\left(\int_{b^{-1}\left(\rho^{-1}(s)\right)}^{s} k^{q}\left(x, \rho^{-1}(s)\right) w^{q}(x) d x\right)^{\frac{1}{q}}\left(\int_{a(s)}^{\rho^{-1}(s)} v^{p^{\prime}}(y) d y\right)^{\frac{1}{p^{\prime}}} \\
& =2^{1 / q} A_{0}\left(b^{-1}\left(\rho^{-1}(s)\right), s\right) \leq \sup _{t>0} \sup _{b^{-1}(a(t)) \leq s \leq t} \mathcal{A}_{0}(s, t) .
\end{aligned}
$$

On the strength of (4.1) it implies $\mathcal{A}_{\rho}^{-} \ll\|\mathcal{K}\|_{L_{p} \rightarrow L_{q}}$. Analogously,

$$
\begin{aligned}
\mathcal{A}_{\sigma}^{+}(t) & \stackrel{(3.1)}{=} 2^{1 / p^{\prime}}\left(\int_{\delta^{+}(t)} w^{q}(x) d x\right)^{\frac{1}{q}}\left(\int_{\Delta^{+}(t)} k^{p^{\prime}}(t, y) v^{p^{\prime}}(y) d y\right)^{\frac{1}{p^{\prime}}} \\
& =2^{1 / p^{\prime}} A_{1}\left(t, a^{-1}(\sigma(t))\right) \leq \sup _{t>0} \sup _{b^{-1}(a(t)) \leq s \leq t} \mathcal{A}_{1}(s, t)
\end{aligned}
$$

implies $\mathcal{A}_{\sigma}^{+} \ll\|\mathcal{K}\|_{L_{p} \rightarrow L_{q}}$, and the lower estimate in (3.3) is proved.

The upper bound. For the opposite estimate we put $\tau_{0}:=\rho(a(t))$ and write

$$
\begin{aligned}
& \sup _{b^{-1}(a(t)) \leq s \leq t} \mathcal{A}_{0}(s, t) \leq \sup _{b^{-1}(a(t)) \leq s \leq \rho(a(t))<t} \mathcal{A}_{0}(s, t)+\sup _{\rho(a(t)) \leq s \leq t} \mathcal{A}_{0}(s, t) \\
& \leq \sup _{b^{-1}\left(\rho^{-1}\left(\tau_{0}\right)\right) \leq s \leq \tau_{0}}\left(\int_{s}^{a^{-1}\left(\rho^{-1}\left(\tau_{0}\right)\right)} k^{q}(x, b(s)) w^{q}(x) d x\right)^{\frac{1}{q}}\left(\int_{\rho^{-1}\left(\tau_{0}\right)}^{b(s)} v^{p^{\prime}}(y) d y\right)^{\frac{1}{p^{\prime}}} \\
& \quad+\sup _{\rho(a(t)) \leq s \leq t}\left(\int_{s}^{a^{-1}\left(\rho^{-1}(s)\right)} k^{q}(x, b(s)) w^{q}(x) d x\right)^{\frac{1}{q}}\left(\int_{a(s)}^{b(s)} v^{p^{\prime}}(y) d y\right)^{\frac{1}{p^{\prime}}} \\
& =: H_{1}\left(\tau_{0}\right)+H_{2}(t) .
\end{aligned}
$$

Indeed, if $b^{-1}(a(t)) \leq s \leq \rho(a(t))<t$ that is $b^{-1}\left(\rho^{-1}\left(\tau_{0}\right)\right) \leq s \leq \tau_{0}$ then $(s, t)=\left(s, a^{-1}\left(\rho^{-1}\left(\tau_{0}\right)\right)\right)$ and $(a(t), b(s))=\left(\rho^{-1}\left(\tau_{0}\right), b(s)\right)$. If $\rho(a(t)) \leq s \leq$ $t$, then $(s, t) \subset\left(s, a^{-1}\left(\rho^{-1}(s)\right)\right)$ and $(a(t), b(s)) \subset(a(s), b(s))$. 
To estimate $H_{1}\left(\tau_{0}\right)$ we use (1.3) with $y=\rho^{-1}\left(\tau_{0}\right)$ and $z=s$, that is since $s \leq x$ and $a(x) \leq \rho^{-1}\left(\tau_{0}\right) \leq b(s)$ then $k(x, b(s)) \ll k\left(x, \rho^{-1}\left(\tau_{0}\right)\right)$. Therefore,

$$
\begin{aligned}
H_{1}\left(\tau_{0}\right) & \ll\left(\int_{b^{-1}\left(\rho^{-1}\left(\tau_{0}\right)\right)}^{a^{-1}\left(\rho^{-1}\left(\tau_{0}\right)\right)} k^{q}\left(x, \rho^{-1}\left(\tau_{0}\right)\right) w^{q}(x) d x\right)^{\frac{1}{q}}\left(\int_{\rho^{-1}\left(\tau_{0}\right)}^{b\left(\tau_{0}\right)} v^{p^{\prime}}(y) d y\right)^{\frac{1}{p^{\prime}}} \\
& =\left(\int_{b^{-1}(z)}^{\left.a^{-1}(z)\right)} k^{q}(x, z) w^{q}(x) d x\right)^{\frac{1}{q}}\left(\int_{z}^{b(\rho(z))} v^{p^{\prime}}(y) d y\right)^{\frac{1}{p^{\prime}}}=\mathcal{A}_{\rho}^{+}(z) \leq \mathcal{A}_{\rho}^{+} .
\end{aligned}
$$

Since $s \leq x$ and $a(x) \leq \rho^{-1}(s) \leq b(s)$ in $H_{2}(t)$ we obtain by using (1.3) with $z=s$ and $y=\rho^{-1}(s)$

$$
\begin{aligned}
H_{2}(t) & \ll \sup _{\rho(a(t)) \leq s \leq t}\left(\int_{s}^{a^{-1}\left(\rho^{-1}(s)\right)} k^{q}\left(x, \rho^{-1}(s)\right) w^{q}(x) d x\right)^{\frac{1}{q}}\left(\int_{a(s)}^{b(s)} v^{p^{\prime}}(y) d y\right)^{\frac{1}{p^{\prime}}} \\
& =\sup _{a(t) \leq z \leq \rho^{-1}(t)}\left(\int_{\rho(z)}^{a^{-1}(z)} k^{q}(x, z) w^{q}(x) d x\right)^{\frac{1}{q}}\left(\int_{a(\rho(z))}^{b(\rho(z))} v^{p^{\prime}}(y) d y\right)^{\frac{1}{p^{\prime}}} \leq \mathcal{A}_{\rho} .
\end{aligned}
$$

Thus,

$$
\sup _{t>0} \sup _{b^{-1}(a(t)) \leq s \leq t} \mathcal{A}_{0}(s, t) \ll \mathcal{A}_{\rho} .
$$

Analogously, we put $\tau_{1}:=\sigma^{-1}(a(t))$ and write

$$
\begin{aligned}
& \sup _{b^{-1}(a(t)) \leq s \leq t} \mathcal{A}_{1}(s, t) \leq \sup _{b^{-1}(a(t)) \leq s \leq \sigma^{-1}(a(t))<t} \mathcal{A}_{1}(s, t)+\sup _{\sigma^{-1}(a(t)) \leq s \leq t} \mathcal{A}_{1}(s, t) \\
& \leq \sup _{b^{-1}\left(\sigma\left(\tau_{1}\right)\right) \leq s \leq \tau_{1}}\left(\int_{s}^{a^{-1}\left(\sigma\left(\tau_{1}\right)\right)} w^{q}(x) d x\right)^{\frac{1}{q}}\left(\int_{\sigma\left(\tau_{1}\right)}^{b(s)} k^{p^{\prime}}(s, y) v^{p^{\prime}}(y) d y\right)^{\frac{1}{p^{\prime}}} \\
& \quad+\sup _{\sigma^{-1}(a(t)) \leq s \leq t}\left(\int_{s}^{a^{-1}(\sigma(s))} w^{q}(x) d x\right)^{\frac{1}{q}}\left(\int_{a(s)}^{b(s)} k^{p^{\prime}}(s, y) v^{p^{\prime}}(y) d y\right)^{\frac{1}{p^{\prime}}} \\
& =: H_{3}\left(\tau_{1}\right)+H_{4}(t) .
\end{aligned}
$$

Obviously, $H_{4}(t) \leq \sup _{\sigma^{-1}(a(t)) \leq s \leq t} \mathcal{A}_{\sigma}^{+}(s) \leq \mathcal{A}_{\sigma}^{+}$. For $H_{3}\left(\tau_{1}\right)$ we apply (1.3) with $z=s \leq \tau_{1}=x$ and $a\left(\tau_{1}\right)<\sigma\left(\tau_{1}\right) \leq y \leq b(s)$ :

$$
\begin{aligned}
H_{3}\left(\tau_{1}\right) & \ll \sup _{b^{-1}\left(\sigma\left(\tau_{1}\right)\right) \leq s \leq \tau_{1}}\left(\int_{s}^{a^{-1}\left(\sigma\left(\tau_{1}\right)\right)} w^{q}(x) d x\right)^{\frac{1}{q}}\left(\int_{\sigma\left(\tau_{1}\right)}^{b(s)} k^{p^{\prime}}\left(\tau_{1}, y\right) v^{p^{\prime}}(y) d y\right)^{\frac{1}{p^{\prime}}} \\
& \leq \mathcal{A}_{\sigma}\left(\tau_{1}\right) \leq \mathcal{A}_{\sigma} .
\end{aligned}
$$


Thus,

$$
\sup _{t>0} \sup _{b^{-1}(a(t)) \leq s \leq t} \mathcal{A}_{1}(s, t) \ll \mathcal{A}_{\sigma} .
$$

Combining (4.1), (4.2) and (4.3), we obtain the upper estimate in (3.3). Necessary and sufficient compactness conditions for $1<p \leq q<\infty$ follow from Theorem 1.1.

(b) Now we consider the case $1<q<p<\infty$. We prove first the upper estimate in (3.4). To this end we take a point sequence $\left\{\xi_{k}\right\}_{k \in \mathbb{Z}} \subset(0, \infty)$ such that $\xi_{0}=1, \xi_{k}=\left(a^{-1} \circ b\right)^{k}(1), k \in \mathbb{Z}$, and put

$\eta_{k}=a\left(\xi_{k}\right)=b\left(\xi_{k-1}\right), \quad \Delta_{k}=\left[\xi_{k}, \xi_{k+1}\right), \quad \delta_{k}=\left[\eta_{k}, \eta_{k+1}\right), \quad k \in \mathbb{Z}$.

Breaking the semiaxis $(0, \infty)$ by points $\left\{\xi_{k}\right\}_{k \in \mathbb{Z}}$ we decompose the operator $\mathcal{K}$ into the sum

$$
\mathcal{K}=\mathcal{T}+\mathcal{S}
$$

of block-diagonal operators $\mathcal{T}$ and $\mathcal{S}$ such that

$$
\mathcal{T}=\sum_{k \in \mathbb{Z}} T_{k}, \quad \mathcal{S}=\sum_{k \in \mathbb{Z}} S_{k},
$$

where

$$
\begin{array}{ll}
T_{k} f(x)=w(x) \int_{a(x)}^{a\left(\xi_{k+1}\right)} k(x, y) f(y) v(y) d y, & T_{k}: L_{p}\left(\delta_{k}\right) \rightarrow L_{q}\left(\Delta_{k}\right), \\
S_{k} f(x)=w(x) \int_{b\left(\xi_{k}\right)}^{b(x)} k(x, y) f(y) v(y) d y, & S_{k}: L_{p}\left(\delta_{k+1}\right) \rightarrow L_{q}\left(\Delta_{k}\right) .
\end{array}
$$

Kernels $k(x, y)$ of the operators $T_{k}$ and $S_{k}$ satisfy the condition (1.3) for $z \leq x, x \in\left[\xi_{k}, \xi_{k+1}\right]$ and

$$
a(x) \leq y \leq b\left(\xi_{k}\right), \quad b\left(\xi_{k}\right) \leq y \leq b(z),
$$

respectively. Since $\sqcup_{k \in \mathbb{Z}}\left[\xi_{k}, \xi_{k+1}\right)=(0, \infty)$ it follows from $(4.4)-(4.5)$ that

$$
\|\mathcal{K} f\|_{q}^{q}=\sum_{k \in \mathbb{Z}}\|\mathcal{K} f\|_{L_{q}\left(\Delta_{k}\right)}^{q} \approx \sum_{k \in \mathbb{Z}}\left\|T_{k} f\right\|_{L_{q}\left(\Delta_{k}\right)}^{q}+\sum_{k \in \mathbb{Z}}\left\|S_{k} f\right\|_{L_{q}\left(\Delta_{k}\right)}^{q} .
$$

To estimate a norm of the operator $S_{k}$ we take into account two key points $s_{\rho}:=b^{-1}\left(\rho^{-1}\left(\xi_{k+1}\right)\right), s_{\sigma}:=\sigma^{-1}\left(b\left(\xi_{k}\right)\right)=\sigma^{-1}\left(a\left(\xi_{k+1}\right)\right)$ and consider three 
only possible variants:
(i) $s_{\rho}<s_{\sigma}$,
(ii) $s_{\rho}=s_{\sigma}$,
(iii) $s_{\rho}>s_{\sigma}$.

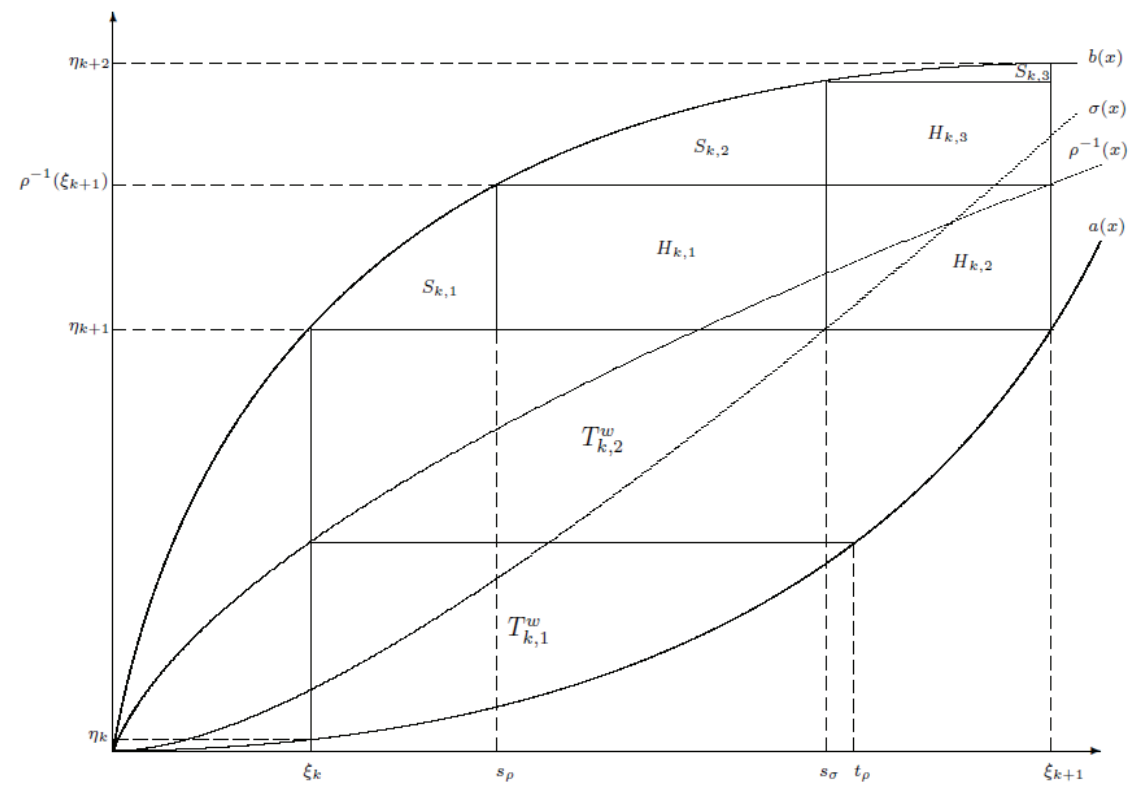

Figure 1. Case (i)

In the case (i) we have

$$
S_{k} f(x)=\sum_{i=1}^{3} S_{k, i} f(x)+\sum_{i=1}^{3} H_{k, i} f(x),
$$

where

$$
\begin{gathered}
S_{k, 1} f(x)=\chi_{\left[\xi_{k}, s_{\rho}\right]}(x) S_{k} f(x), S_{k, 1}: L_{p}\left(b\left(\xi_{k}\right), b\left(s_{\rho}\right)\right) \rightarrow L_{q}\left(\xi_{k}, s_{\rho}\right), \\
H_{k, 1} f(x)=\chi_{\left[s_{\rho}, s_{\sigma}\right]}(x) S_{k}\left(f \chi_{\left[b\left(\xi_{k}\right), b\left(s_{\rho}\right)\right]}\right)(x), H_{k, 1}: L_{p}\left(b\left(\xi_{k}\right), b\left(s_{\rho}\right)\right) \rightarrow L_{q}\left(s_{\rho}, s_{\sigma}\right), \\
S_{k, 2} f(x)=\chi_{\left[s_{\rho}, s_{\sigma}\right]}(x) S_{k}\left(f \chi_{\left[b\left(s_{\rho}\right), b\left(s_{\sigma}\right)\right]}\right)(x), S_{k, 2}: L_{p}\left(b\left(s_{\rho}\right), b\left(s_{\sigma}\right)\right) \rightarrow L_{q}\left(s_{\rho}, s_{\sigma}\right), \\
H_{k, 2} f(x)=\chi_{\left[s_{\sigma}, \xi_{k+1}\right]}(x) S_{k}\left(f \chi_{\left[b\left(\xi_{k}\right), b\left(s_{\rho}\right)\right]}\right)(x), H_{k, 2}: L_{p}\left(b\left(\xi_{k}\right), b\left(s_{\rho}\right)\right) \rightarrow L_{q}\left(s_{\sigma}, \xi_{k+1}\right), \\
H_{k, 3} f(x)=\chi_{\left[s_{\sigma}, \xi_{k+1}\right]}(x) S_{k}\left(f \chi_{\left[b\left(s_{\rho}\right), b\left(s_{\sigma}\right)\right]}\right)(x), H_{k, 3}: L_{p}\left(b\left(s_{\rho}\right), b\left(s_{\sigma}\right)\right) \rightarrow L_{q}\left(s_{\sigma}, \xi_{k+1}\right), \\
S_{k, 3} f(x)=\chi_{\left[s_{\sigma}, \xi_{k+1}\right]}(x) S_{k}\left(f \chi_{\left[b\left(s_{\sigma}\right), b\left(\xi_{k+1}\right)\right]}\right)(x), S_{k, 3}: L_{p}\left(b\left(s_{\sigma}\right), b\left(\xi_{k+1}\right)\right) \rightarrow L_{q}\left(s_{\sigma}, \xi_{k+1}\right) .
\end{gathered}
$$


Applying Corollary 2.1 with $K_{b}=S_{k, 1}, c=\xi_{k}, d=s_{\rho}$ and Lemma 2.2 we obtain

$$
\begin{aligned}
\left\|S_{k, 1}\right\|^{r}:= & \left\|S_{k, 1}\right\|_{L_{p}\left(b\left(\xi_{k}\right), b\left(s_{\rho}\right)\right) \rightarrow L_{q}\left(\xi_{k}, s_{\rho}\right)}^{r} \\
\approx & B_{b, 0}^{r}+B_{b, 1}^{r} \stackrel{(2.11)}{\ll} B_{b, 0}^{r}+\mathbb{B}_{b, 1}^{r} \\
= & \int_{b\left(\xi_{k}\right)}^{b\left(s_{\rho}\right)}\left(\int_{b^{-1}(t)}^{s_{\rho}} k^{q}(x, t) w^{q}(x) d x\right)^{\frac{r}{q}}\left(\int_{b\left(\xi_{k}\right)}^{t} v^{p^{\prime}}(y) d y\right)^{\frac{r}{q^{\prime}}} v^{p^{\prime}}(t) d t \\
& +\int_{\xi_{k}}^{s_{\rho}}\left(\int_{\xi_{k}}^{t}\left[\int_{b\left(\xi_{k}\right)}^{b(x)} k^{p^{\prime}}(x, y) v^{p^{\prime}}(y) d y\right]^{q} w^{q}(x) d x\right)^{\frac{r}{p}} \times \\
& \times\left[\int_{b\left(\xi_{k}\right)}^{b(t)} k^{p^{\prime}}(t, y) v^{p^{\prime}}(y) d y\right]^{q-\frac{r}{p}} w^{q}(t) d t .
\end{aligned}
$$

Since $t \geq b\left(\xi_{k}\right)>a\left(s_{\rho}\right)$ in $B_{b, 0}$ then $\left[b^{-1}(t), s_{\rho}\right] \subset\left[b^{-1}(t), a^{-1}(t)\right]$, and $\left[b\left(\xi_{k}\right), t\right] \subseteq[a(\rho(t)), t]$ because of $t \leq b\left(s_{\rho}\right)=\rho^{-1}\left(\xi_{k+1}\right) \Longrightarrow \rho(t) \leq \xi_{k+1}$ $\Longrightarrow a(\rho(t)) \leq a\left(\xi_{k+1}\right)=b\left(\xi_{k}\right)$. Therefore

$$
\begin{aligned}
B_{b, 0}^{r} & \leq \int_{b\left(\xi_{k}\right)}^{b\left(s_{\rho}\right)}\left(\int_{\Theta(t)} k^{q}(x, t) w^{q}(x) d x\right)^{\frac{r}{q}}\left(\int_{\vartheta^{-}(t)} v^{p^{\prime}}(y) d y\right)^{\frac{r}{q^{\prime}}} v^{p^{\prime}}(t) d t \\
(4.10) & \leq \int_{\delta_{k+1}} \mathcal{B}_{\rho}^{-}(t) d t .
\end{aligned}
$$

To estimate $\mathbb{B}_{b, 1}$ note that in view of $(1.3)$ we have $k(x, y) \ll k(t, y)$, where $x \leq t, a(t)<b\left(\xi_{k}\right) \leq y \leq b(x)$ and

$$
\mathbb{B}_{b, 1}^{r} \ll \int_{\xi_{k}}^{s_{\rho}}\left(\int_{\xi_{k}}^{t} w^{q}(x) d x\right)^{\frac{r}{p}}\left(\int_{b\left(\xi_{k}\right)}^{b(t)} k^{p^{\prime}}(t, y) v^{p^{\prime}}(y) d y\right)^{\frac{r}{p^{\prime}}} w^{q}(t) d t .
$$

Here $\left[\xi_{k}, t\right] \subset\left[b^{-1}(\sigma(t)), t\right]$ because $t \leq s_{\rho}<s_{\sigma}=\sigma^{-1}\left(b\left(\xi_{k}\right)\right) \Longrightarrow$ $\sigma(t)<b\left(\xi_{k}\right) \Longrightarrow b^{-1}(\sigma(t))<\xi_{k}$. Obviously, $\left[b\left(\xi_{k}\right), b(t)\right] \subset[a(t), b(t)]$. Therefore

$$
\begin{aligned}
\mathbb{B}_{b, 1}^{r} & \ll \int_{\xi_{k}}^{s_{\rho}}\left(\int_{\delta^{-}(t)} w^{q}(x) d x\right)^{\frac{r}{p}}\left(\int_{\Delta(t)} k^{p^{\prime}}(t, y) v^{p^{\prime}}(y) d y\right)^{\frac{r}{p^{\prime}}} w^{q}(t) d t \\
(4.11) & \leq \int_{\Delta_{k}} \mathcal{B}_{\sigma}^{-}(t) d t .
\end{aligned}
$$


Next, we decompose the operator $H_{k, 1}$ into a sum by using (1.3) with $z=s_{\rho} \leq x \leq s_{\sigma}, a(x)<b\left(\xi_{k}\right) \leq y \leq b\left(s_{\rho}\right)=b(z):$

$$
\begin{aligned}
H_{k, 1} f(x) & =w(x) k\left(x, b\left(s_{\rho}\right)\right) \int_{b\left(\xi_{k}\right)}^{b\left(s_{\rho}\right)} f(y) v(y) d y+w(x) \int_{b\left(\xi_{k}\right)}^{b\left(s_{\rho}\right)} k\left(s_{\rho}, y\right) f(y) v(y) d y \\
& =: H_{k, 1}^{w} f(x)+H_{k, 1}^{v} f(x) .
\end{aligned}
$$

By Hölder's inequality and (1.3)

$$
\begin{aligned}
\left\|H_{k, 1}^{w}\right\|^{r} & =\left(\int_{s_{\rho}}^{s_{\sigma}} k^{q}\left(x, b\left(s_{\rho}\right)\right) w^{q}(x) d x\right)^{\frac{r}{q}}\left(\int_{b\left(\xi_{k}\right)}^{b\left(s_{\rho}\right)} v^{p^{\prime}}(y) d y\right)^{\frac{r}{p^{\prime}}} \\
& \stackrel{(1.3)}{\ll} \int_{b\left(\xi_{k}\right)}^{b\left(s_{\rho}\right)}\left(\int_{b\left(\xi_{k}\right)}^{t} v^{p^{\prime}}(y) d y\right)^{\frac{r}{q}}\left(\int_{b^{-1}(t)}^{s_{\sigma}} k^{q}(x, t) w^{q}(x) d x\right)^{\frac{r}{q}} v^{p^{\prime}}(t) d t \\
& <\int_{b\left(\xi_{k}\right)}^{b\left(s_{\rho}\right)}\left(\int_{\Theta(t)} k^{q}(x, t) w^{q}(x) d x\right)^{\frac{r}{q}}\left(\int_{\vartheta^{-}(t)} v^{p^{\prime}}(y) d y\right)^{\frac{r}{q^{\prime}}} v^{p^{\prime}}(t) d t \\
(4.12) & \leq \int_{\delta_{k+1}} \mathcal{B}_{\rho}^{-}(t) d t
\end{aligned}
$$

because $a\left(s_{\sigma}\right)<t$ and $t \leq b\left(s_{\rho}\right)=\rho^{-1}\left(\xi_{k+1}\right) \Longrightarrow \rho(t) \leq \xi_{k+1} \Longrightarrow$ $a(\rho(t)) \leq a\left(\xi_{k+1}\right)=b\left(\xi_{k}\right)$. Analogously,

$$
\begin{aligned}
&\left\|H_{k, 1}^{v}\right\|^{r}=\left(\int_{s_{\rho}}^{s_{\sigma}} w^{q}(x) d x\right)^{\frac{r}{q}}\left(\int_{b\left(\xi_{k}\right)}^{b\left(s_{\rho}\right)} k^{p^{\prime}}\left(s_{\rho}, y\right) v^{p^{\prime}}(y) d y\right)^{\frac{r}{p^{\prime}}} \\
& \ll \int_{s_{\rho}}^{s_{\sigma}}\left(\int_{s_{\rho}}^{t} w^{q}(x) d x\right)^{\frac{r}{p}}\left(\int_{b\left(\xi_{k}\right)}^{b(t)} k^{p^{\prime}}(t, y) v^{p^{\prime}}(y) d y\right)^{\frac{r}{p^{\prime}}} w^{q}(t) d t \\
&<\int_{s_{\rho}}^{s_{\sigma}}\left(\int_{\delta^{-}(t)} w^{q}(x) d x\right)^{\frac{r}{p}}\left(\int_{\Delta(t)} k^{p^{\prime}}(t, y) v^{p^{\prime}}(y) d y\right)^{\frac{r}{p^{\prime}}} w^{q}(t) d t \\
&(4.13) \leq \int_{\Delta_{k}} \mathcal{B}_{\sigma}^{-}(t) d t
\end{aligned}
$$

on the strength of $a(t)<a\left(\xi_{k+1}\right)=b\left(\xi_{k}\right)$ and $t \leq s_{\sigma} \Longrightarrow \sigma(t) \leq b\left(\xi_{k}\right)$ $\Longrightarrow b^{-1}(\sigma(t)) \leq \xi_{k}<s_{\rho}$. For $S_{k, 2}$ we use the result of Corollary 2.1 with 
$c=s_{\rho}, d=s_{\sigma}$ and Lemma 2.2:

$$
\begin{aligned}
\left\|S_{k, 2}\right\|^{r}:= & \left\|S_{k, 2}\right\|_{L_{p}\left(b\left(s_{\rho}\right), b\left(s_{\sigma}\right)\right) \rightarrow L_{q}\left(s_{\rho}, s_{\sigma}\right)}^{r} \\
\approx & B_{b, 0}^{r}+B_{b, 1}^{r} \stackrel{(2.10),(2.11)}{\ll} \mathcal{B}_{b, 0}^{r}+\mathbb{B}_{b, 1}^{r} \\
= & \int_{b\left(s_{\rho}\right)}^{b\left(s_{\sigma}\right)}\left(\int_{t}^{b\left(s_{\sigma}\right)}\left[\int_{b^{-1}(y)}^{s_{\sigma}} k^{q}(x, y) w^{q}(x) d x\right]^{p^{\prime}} v^{p^{\prime}}(y) d y\right)^{\frac{r}{q^{\prime}}} \times \\
& \times\left[\int_{b^{-1}(t)}^{s_{\sigma}} k^{q}(x, t) w^{q}(x) d x\right]^{p^{\prime}-\frac{r}{q^{\prime}}} v^{p^{\prime}}(t) d t \\
& +\int_{s_{\rho}}^{s_{\sigma}}\left(\int_{s_{\rho}}^{t}\left[\int_{b\left(s_{\rho}\right)}^{b(x)} k^{p^{\prime}}(x, y) v^{p^{\prime}}(y) d y\right]^{q} w^{q}(x) d x\right)^{\frac{r}{p}} \times \\
& \times\left[\int_{b\left(s_{\rho}\right)}^{b(t)} k^{p^{\prime}}(t, y) v^{p^{\prime}}(y) d y\right]^{q-\frac{r}{p}} w^{q}(t) d t .
\end{aligned}
$$

Since $a(x)<b\left(\xi_{k}\right)<b\left(s_{\rho}\right) \leq t \leq y=b(z)$ with $z=b^{-1}(y) \leq x$ in $\mathcal{B}_{b, 0}$ then $k(x, y)=k(x, b(z)) \ll k(x, t)$. Therefore,

$$
\mathcal{B}_{b, 0}^{r} \ll \int_{b\left(s_{\rho}\right)}^{b\left(s_{\sigma}\right)}\left(\int_{t}^{b\left(s_{\sigma}\right)} v^{p^{\prime}}(y) d y\right)^{\frac{r}{q^{\prime}}}\left(\int_{b^{-1}(t)}^{s_{\sigma}} k^{q}(x, t) w^{q}(x) d x\right)^{\frac{r}{q}} v^{p^{\prime}}(t) d t .
$$

Since $s_{\sigma}<\xi_{k+1}$ then $a\left(s_{\sigma}\right)<\rho^{-1}\left(s_{\sigma}\right)<\rho^{-1}\left(\xi_{k+1}\right)=b\left(s_{\rho}\right) \leq t \Longrightarrow s_{\sigma}<$ $a^{-1}(t)$ and $s_{\sigma}<\rho(t) \Longrightarrow b\left(s_{\sigma}\right)<b(\rho(t))$. Thus, $\left[t, b\left(s_{\sigma}\right)\right] \subset[t, b(\rho(t))]$, $\left[b^{-1}(t), s_{\sigma}\right] \subset\left[b^{-1}(t), a^{-1}(t)\right]$ and

$$
\begin{aligned}
\mathcal{B}_{b, 0}^{r} & \ll \int_{b\left(s_{\rho}\right)}^{b\left(s_{\sigma}\right)}\left(\int_{\Theta(t)} k^{q}(x, t) w^{q}(x) d x\right)^{\frac{r}{q}}\left(\int_{\vartheta^{+}(t)} v^{p^{\prime}}(y) d y\right)^{\frac{r}{q}} v^{p^{\prime}}(t) d t \\
(4.15) & \leq \int_{\delta_{k+1}} \mathcal{B}_{\rho}^{+}(t) d t .
\end{aligned}
$$

In $\mathbb{B}_{b, 1}$ since $x \leq t, a(t)<b\left(\xi_{k}\right)<b\left(s_{\rho}\right) \leq y \leq b(x)$ we have $k(x, y) \ll k(t, y)$. Therefore, in view of $\xi_{k}<s_{\rho} \leq t \leq s_{\sigma}<\xi_{k+1} \Longrightarrow$ $\sigma(t) \leq b\left(\xi_{k}\right) \Longrightarrow b^{-1}(\sigma(t)) \leq \xi_{k}<s_{\rho}$ and $a(t)<b\left(\xi_{k}\right)$

$$
\mathbb{B}_{b, 1}^{r} \ll \int_{s_{\rho}}^{s_{\sigma}}\left(\int_{s_{\rho}}^{t} w^{q}(x) d x\right)^{\frac{r}{p}}\left(\int_{b\left(s_{\rho}\right)}^{b(t)} k^{p^{\prime}}(t, y) v^{p^{\prime}}(y) d y\right)^{\frac{r}{p^{\prime}}} w^{q}(t) d t
$$




$$
\begin{aligned}
& <\int_{s_{\rho}}^{s_{\sigma}}\left(\int_{\delta^{-}(t)} w^{q}(x) d x\right)^{\frac{r}{p}}\left(\int_{\Delta(t)} k^{p^{\prime}}(t, y) v^{p^{\prime}}(y) d y\right)^{\frac{r}{p^{\prime}}} w^{q}(t) d t \\
& \leq \int_{\Delta_{k}} \mathcal{B}_{\sigma}^{-}(t) d t .
\end{aligned}
$$

By using (1.3) with $z=s_{\rho}<x \leq \xi_{k+1}, a(x)<b\left(\xi_{k}\right) \leq y \leq b\left(s_{\rho}\right)=b(z)$ we decompose $H_{k, 2}$ into the sum

$$
\begin{aligned}
H_{k, 2} f(x) & =w(x) k\left(x, b\left(s_{\rho}\right)\right) \int_{b\left(\xi_{k}\right)}^{b\left(s_{\rho}\right)} f(y) v(y) d y+w(x) \int_{b\left(\xi_{k}\right)}^{b\left(s_{\rho}\right)} k\left(s_{\rho}, y\right) f(y) v(y) d y \\
& =: \quad H_{k, 2}^{w} f(x)+H_{k, 2}^{v} f(x) .
\end{aligned}
$$

Denote $\left\|H_{k, 2}\right\|:=\left\|H_{k, 2}\right\|_{L_{p}\left(b\left(\xi_{k}\right), b\left(s_{\rho}\right)\right) \rightarrow L_{q}\left(s_{\sigma}, \xi_{k+1}\right)}$. By Hölder's inequality and (1.3)

$$
\begin{aligned}
\left\|H_{k, 2}^{w}\right\|^{r} & <\left(\int_{s_{\rho}}^{\xi_{k+1}} k^{q}\left(x, b\left(s_{\rho}\right)\right) w^{q}(x) d x\right)^{\frac{r}{q}}\left(\int_{b\left(\xi_{k}\right)}^{b\left(s_{\rho}\right)} v^{p^{\prime}}(y) d y\right)^{\frac{r}{p^{\prime}}} \\
& \ll \int_{b\left(\xi_{k}\right)}^{b\left(s_{\rho}\right)}\left(\int_{b\left(\xi_{k}\right)}^{t} v^{p^{\prime}}(y) d y\right)^{\frac{r}{q^{\prime}}}\left(\int_{b^{-1}(t)}^{\xi_{k+1}} k^{q}(x, t) w^{q}(x) d x\right)^{\frac{r}{q}} v^{p^{\prime}}(t) d t \\
& <\int_{b\left(\xi_{k}\right)}^{b\left(s_{\rho}\right)}\left(\int_{\Theta(t)} k^{q}(x, t) w^{q}(x) d x\right)^{\frac{r}{q}}\left(\int_{\vartheta^{-}(t)} v^{p^{\prime}}(y) d y\right)^{\frac{r}{q^{\prime}}} v^{p^{\prime}}(t) d t \\
(4.17) & \leq \int_{\delta_{k+1}} \mathcal{B}_{\rho}^{-}(t) d t
\end{aligned}
$$

since $t$ is still not greater then $\rho^{-1}\left(\xi_{k+1}\right)=b\left(s_{\rho}\right)$. Again by Hölder's inequality and (1.3)

$$
\begin{aligned}
\left\|H_{k, 2}^{v}\right\|^{r} & =\left(\int_{s_{\sigma}}^{\xi_{k+1}} w^{q}(x) d x\right)^{\frac{r}{q}}\left(\int_{b\left(\xi_{k}\right)}^{b\left(s_{\rho}\right)} k^{p^{\prime}}\left(s_{\rho}, y\right) v^{p^{\prime}}(y) d y\right)^{\frac{r}{p^{\prime}}} \\
& \ll \int_{s_{\sigma}}^{\xi_{k+1}}\left(\int_{t}^{\xi_{k+1}} w^{q}(x) d x\right)^{\frac{r}{p}}\left(\int_{b\left(\xi_{k}\right)}^{b(t)} k^{p^{\prime}}(t, y) v^{p^{\prime}}(y) d y\right)^{\frac{r}{p^{\prime}}} w^{q}(t) d t \\
& <\int_{s_{\sigma}}^{\xi_{k+1}}\left(\int_{\delta^{+}(t)} w^{q}(x) d x\right)^{\frac{r}{p}}\left(\int_{\Delta(t)} k^{p^{\prime}}(t, y) v^{p^{\prime}}(y) d y\right)^{\frac{r}{p^{\prime}}} w^{q}(t) d t \\
(4.18) & \leq \int_{\Delta_{k}} \mathcal{B}_{\sigma}^{+}(t) d t
\end{aligned}
$$


on the strength of $a(t) \leq b\left(\xi_{k}\right)$ and $s_{\sigma} \leq t \Longrightarrow a\left(\xi_{k+1}\right) \leq \sigma(t) \Longrightarrow$ $\xi_{k+1} \leq a^{-1}(\sigma(t))$. To estimate $S_{k, 3}$ we use again Corollary 2.1 with $c=s_{\sigma}$, $d=\xi_{k+1}$ and Lemma 2.2:

$\left\|S_{k, 3}\right\|^{r}:=\left\|S_{k, 3}\right\|_{L_{p}\left(b\left(s_{\sigma}\right), b\left(\xi_{k+1}\right)\right) \rightarrow L_{q}\left(s_{\sigma}, \xi_{k+1}\right)}^{r}$

$$
\begin{aligned}
& \approx B_{b, 0}^{r}+B_{b, 1}^{r} \\
& \stackrel{(2.10)}{\ll} \mathcal{B}_{b, 0}^{r}+B_{b, 1}^{r} \\
& =\int_{b\left(s_{\sigma}\right)}^{b\left(\xi_{k+1}\right)}\left(\int_{t}^{b\left(\xi_{k+1}\right)}\left[\int_{b^{-1}(y)}^{\xi_{k+1}} k^{q}(x, y) w^{q}(x) d x\right]^{p^{\prime}} v^{p^{\prime}}(y) d y\right)^{\frac{r}{q^{\prime}}} \times \\
& \quad \times\left[\int_{b^{-1}(t)}^{\xi_{k+1}} k^{q}(x, t) w^{q}(x) d x\right]^{p^{\prime}-\frac{r}{q^{\prime}}} v^{p^{\prime}}(t) d t \\
& +\int_{s_{\sigma}}^{\xi_{k+1}}\left(\int_{t}^{\xi_{k+1}} w^{q}(x) d x\right)^{\frac{r}{p^{\prime}}}\left(\int_{b\left(s_{\sigma}\right)}^{b(t)} k^{p^{\prime}}(t, y) v^{p^{\prime}}(y) d y\right)^{\frac{r}{p}} w^{q}(t) d t .
\end{aligned}
$$

As before $k(x, y) \ll k(x, t)$ for $a(x) \leq b\left(\xi_{k}\right)<b\left(s_{\sigma}\right) \leq t \leq y=b(z)$ with $z=b^{-1}(y) \leq x$ in $\mathcal{B}_{b, 0}$, and also $b\left(\xi_{k+1}\right)<b(\rho(t))$ since $\rho^{-1}\left(\xi_{k+1}\right)<t$. Therefore,

$$
\begin{aligned}
\mathcal{B}_{b, 0}^{r} & \ll \int_{b\left(s_{\sigma}\right)}^{b\left(\xi_{k+1}\right)}\left(\int_{t}^{b\left(\xi_{k+1}\right)} v^{p^{\prime}}(y) d y\right)^{\frac{r}{q^{\prime}}}\left(\int_{b^{-1}(t)}^{\xi_{k+1}} k^{q}(x, t) w^{q}(x) d x\right)^{\frac{r}{q}} v^{p^{\prime}}(t) d t \\
& <\int_{b\left(s_{\sigma}\right)}^{b\left(\xi_{k+1}\right)}\left(\int_{\Theta(t)} k^{q}(x, t) w^{q}(x) d x\right)^{\frac{r}{q}}\left(\int_{\vartheta^{+}(t)} v^{p^{\prime}}(y) d y\right)^{\frac{r}{q^{\prime}}} v^{p^{\prime}}(t) d t
\end{aligned}
$$

$$
\leq \int_{\delta_{k+1}} \mathcal{B}_{\rho}^{+}(t) d t
$$

The estimate

$$
\begin{aligned}
B_{b, 1}^{r} & \ll \int_{s_{\sigma}}^{\xi_{k+1}}\left(\int_{\delta^{+}(t)} w^{q}(x) d x\right)^{\frac{r}{p}}\left(\int_{\Delta(t)} k^{p^{\prime}}(t, y) v^{p^{\prime}}(y) d y\right)^{\frac{r}{p^{\prime}}} w^{q}(t) d t \\
& \leq \int_{\Delta_{k}} \mathcal{B}_{\sigma}^{+}(t) d t
\end{aligned}
$$

follows from $a(t) \leq a\left(\xi_{k+1}\right)=b\left(\xi_{k}\right)<b\left(s_{\sigma}\right)$ and $s_{\sigma} \leq t \Longrightarrow a\left(\xi_{k+1}\right) \leq \sigma(t)$ $\Longrightarrow \xi_{k+1} \leq a^{-1}(\sigma(t))$. To estimate the last operator norm $\left\|H_{k, 3}\right\|:=$ 
$\left\|H_{k, 3}\right\|_{L_{p}\left(b\left(s_{\rho}\right), b\left(s_{\sigma}\right)\right) \rightarrow L_{q}\left(s_{\sigma}, \xi_{k+1}\right)}$ we make a decomposition

$$
\begin{aligned}
H_{k, 3} f(x) & =w(x) k\left(x, b\left(s_{\sigma}\right)\right) \int_{b\left(s_{\rho}\right)}^{b\left(s_{\sigma}\right)} f(y) v(y) d y+w(x) \int_{b\left(s_{\rho}\right)}^{b\left(s_{\sigma}\right)} k\left(s_{\sigma}, y\right) f(y) v(y) d y \\
& =: \quad H_{k, 3}^{w} f(x)+H_{k, 3}^{v} f(x)
\end{aligned}
$$

because $k(x, y) \approx k\left(x, b\left(s_{\sigma}\right)\right)+k\left(s_{\sigma}, y\right)$ for $s_{\sigma} \leq x$ and $a(x) \leq y \leq b\left(s_{\sigma}\right)$.

By Hölder's inequality

$$
\begin{aligned}
\left\|H_{k, 3}^{w}\right\|^{r} & =\left(\int_{s_{\sigma}}^{\xi_{k+1}} k^{q}\left(x, b\left(s_{\sigma}\right)\right) w^{q}(x) d x\right)^{\frac{r}{q}}\left(\int_{b\left(s_{\rho}\right)}^{b\left(s_{\sigma}\right)} v^{p^{\prime}}(y) d y\right)^{\frac{r}{p^{\prime}}} \\
& \ll \int_{b\left(s_{\rho}\right)}^{b\left(s_{\sigma}\right)}\left(\int_{t}^{b\left(s_{\sigma}\right)} v^{p^{\prime}}(y) d y\right)^{\frac{r}{q}}\left(\int_{b^{-1}(t)}^{\xi_{k+1}} k^{q}(x, t) w^{q}(x) d x\right)^{\frac{r}{q}} v^{p^{\prime}}(t) d t \\
& <\int_{b\left(s_{\rho}\right)}^{b\left(s_{\sigma}\right)}\left(\int_{\Theta(t)} k^{q}(x, t) w^{q}(x) d x\right)^{\frac{r}{q}}\left(\int_{\vartheta^{+}(t)} v^{p^{\prime}}(y) d y\right)^{\frac{r}{q^{\prime}}} v^{p^{\prime}}(t) d t \\
(4.22) & \leq \int_{\delta_{k+1}} \mathcal{B}_{\rho}^{+}(t) d t
\end{aligned}
$$

since $\rho^{-1}\left(s_{\sigma}\right)<\rho^{-1}\left(\xi_{k+1}\right)=b\left(s_{\rho}\right)<b\left(s_{\sigma}\right) \leq t \Longrightarrow s_{\sigma}<\rho(t) \Longrightarrow$ $b\left(s_{\sigma}\right)<b(\rho(t))$. We have also

$$
\begin{aligned}
\left\|H_{k, 3}^{v}\right\|^{r} & =\left(\int_{s_{\sigma}}^{\xi_{k+1}} w^{q}(x) d x\right)^{\frac{r}{q}}\left(\int_{b\left(s_{\rho}\right)}^{b\left(s_{\sigma}\right)} k^{p^{\prime}}\left(s_{\sigma}, y\right) v^{p^{\prime}}(y) d y\right)^{\frac{r}{p^{\prime}}} \\
& \ll \int_{s_{\sigma}}^{\xi_{k+1}}\left(\int_{t}^{\xi_{k+1}} w^{q}(x) d x\right)^{\frac{r}{p}}\left(\int_{b\left(s_{\rho}\right)}^{b(t)} k^{p^{\prime}}(t, y) v^{p^{\prime}}(y) d y\right)^{\frac{r}{p^{\prime}}} w^{q}(t) d t \\
& <\int_{s_{\sigma}}^{\xi_{k+1}}\left(\int_{\delta^{+}(t)} w^{q}(x) d x\right)^{\frac{r}{p}}\left(\int_{\Delta(t)} k^{p^{\prime}}(t, y) v^{p^{\prime}}(y) d y\right)^{\frac{r}{p^{\prime}}} w^{q}(t) d t \\
(4.23) & \leq \int_{\Delta_{k}} \mathcal{B}_{\sigma}^{+}(t) d t
\end{aligned}
$$

since $s_{\sigma} \leq t \Longrightarrow a\left(\xi_{k+1}\right) \leq \sigma(t) \Longrightarrow \xi_{k+1} \leq a^{-1}(\sigma(t))$ and $a(t) \leq b\left(\xi_{k}\right)<$ $b\left(s_{\sigma}\right)$. Thus, by $(4.8)-(4.23)$ it holds for the case (i) that

$$
\left\|S_{k}\right\|_{L_{p}\left(\delta_{k+1}\right) \rightarrow L_{q}\left(\Delta_{k}\right)}^{r} \ll \int_{\delta_{k+1}} \mathcal{B}_{\rho}(t) d t+\int_{\Delta_{k}} \mathcal{B}_{\sigma}(t) d t .
$$




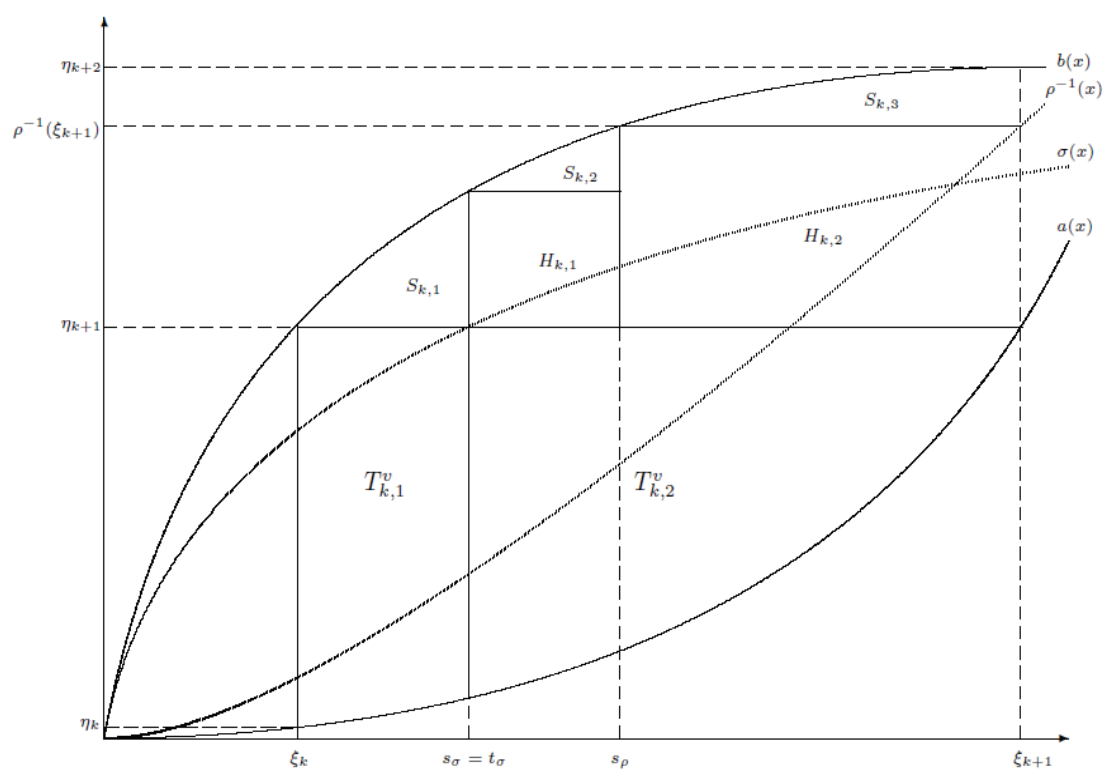

Figure 2. Case (iii)

In the case (iii) we have

$$
S_{k} f(x)=\sum_{i=1}^{3} S_{k, i} f(x)+\sum_{i=1}^{2} H_{k, i} f(x),
$$

where

$$
\begin{gathered}
S_{k, 1} f(x)=\chi_{\left[\xi_{k}, s_{\sigma}\right]}(x) S_{k} f(x), S_{k, 1}: L_{p}\left(b\left(\xi_{k}\right), b\left(s_{\sigma}\right)\right) \rightarrow L_{q}\left(\xi_{k}, s_{\sigma}\right), \\
H_{k, 1} f(x)=\chi_{\left[s_{\sigma}, s_{\rho}\right]}(x) S_{k}\left(f \chi_{\left[b\left(\xi_{k}\right), b\left(s_{\sigma}\right)\right]}\right)(x), H_{k, 1}: L_{p}\left(b\left(\xi_{k}\right), b\left(s_{\sigma}\right)\right) \rightarrow L_{q}\left(s_{\sigma}, s_{\rho}\right), \\
S_{k, 2} f(x)=\chi_{\left[s_{\sigma}, s_{\rho}\right]}(x) S_{k}\left(f \chi_{\left[b\left(s_{\sigma}\right), b\left(s_{\rho}\right)\right]}\right)(x), S_{k, 2}: L_{p}\left(b\left(s_{\sigma}\right), b\left(s_{\rho}\right)\right) \rightarrow L_{q}\left(s_{\sigma}, s_{\rho}\right), \\
H_{k, 2} f(x)=\chi_{\left[s_{\rho}, \xi_{k+1}\right]}(x) S_{k}\left(f \chi_{\left[b\left(\xi_{k}\right), b\left(s_{\rho}\right)\right]}\right)(x), H_{k, 2}: L_{p}\left(b\left(\xi_{k}\right), b\left(s_{\rho}\right)\right) \rightarrow L_{q}\left(s_{\rho}, \xi_{k+1}\right), \\
S_{k, 3} f(x)=\chi_{\left[s_{\rho}, \xi_{k+1}\right]}(x) S_{k}\left(f \chi_{\left[b\left(s_{\rho}\right), b\left(\xi_{k+1}\right)\right]}\right)(x), S_{k, 3}: L_{p}\left(b\left(s_{\rho}\right), b\left(\xi_{k+1}\right)\right) \rightarrow L_{q}\left(s_{\rho}, \xi_{k+1}\right) .
\end{gathered}
$$

The estimate

$$
\left\|S_{k, 1}\right\|^{r}:=\left\|S_{k, 1}\right\|_{L_{p}\left(b\left(\xi_{k}\right), b\left(s_{\sigma}\right)\right) \rightarrow L_{q}\left(\xi_{k}, s_{\sigma}\right)}^{r} \ll \int_{\delta_{k+1}} \mathcal{B}_{\rho}^{-}(t) d t+\int_{\Delta_{k}} \mathcal{B}_{\sigma}^{-}(t) d t
$$


can be obtained analogously to the case (i). The next operator $H_{k, 1}$ should be decomposed by (1.3) with $z=s_{\sigma} \leq x \leq s_{\rho}, a(x)<b\left(\xi_{k}\right) \leq y \leq b\left(s_{\sigma}\right)=$ $b(z)$ into the sum

$$
\begin{aligned}
H_{k, 1} f(x) & =w(x) k\left(x, b\left(s_{\sigma}\right)\right) \int_{b\left(\xi_{k}\right)}^{b\left(s_{\sigma}\right)} f(y) v(y) d y+w(x) \int_{b\left(\xi_{k}\right)}^{b\left(s_{\sigma}\right)} k\left(s_{\sigma}, y\right) f(y) v(y) d y \\
& =: H_{k, 1}^{w} f(x)+H_{k, 1}^{v} f(x) .
\end{aligned}
$$

Putting $\left\|H_{k, 1}\right\|:=\left\|H_{k, 1}\right\|_{L_{p}\left(b\left(\xi_{k}\right), b\left(s_{\sigma}\right)\right) \rightarrow L_{q}\left(s_{\sigma}, s_{\rho}\right)}$, by Hölder's inequality and (1.3) we obtain

$$
\begin{aligned}
\left\|H_{k, 1}^{w}\right\|^{r} & =\left(\int_{s_{\sigma}}^{s_{\rho}} k^{q}\left(x, b\left(s_{\sigma}\right)\right) w^{q}(x) d x\right)^{\frac{r}{q}}\left(\int_{b\left(\xi_{k}\right)}^{b\left(s_{\sigma}\right)} v^{p^{\prime}}(y) d y\right)^{\frac{r}{p^{\prime}}} \\
& \ll \int_{b\left(\xi_{k}\right)}^{b\left(s_{\sigma}\right)}\left(\int_{b\left(\xi_{k}\right)}^{t} v^{p^{\prime}}(y) d y\right)^{\frac{r}{q^{\prime}}}\left(\int_{b^{-1}(t)}^{s_{\rho}} k^{q}(x, t) w^{q}(x) d x\right)^{\frac{r}{q}} v^{p^{\prime}}(t) d t \\
& <\int_{b\left(\xi_{k}\right)}^{b\left(s_{\sigma}\right)}\left(\int_{\Theta(t)} k^{q}(x, t) w^{q}(x) d x\right)^{\frac{r}{q}}\left(\int_{\vartheta-(t)} v^{p^{\prime}}(y) d y\right)^{\frac{r}{q^{\prime}}} v^{p^{\prime}}(t) d t \\
& \leq \int_{\delta_{k+1}} \mathcal{B}_{\rho}^{-}(t) d t \\
\text { since } a\left(s_{\rho}\right)<t & <\operatorname{and} t<b\left(s_{\rho}\right)=\rho^{-1}\left(\xi_{k+1}\right) \Longrightarrow \rho(t)<\xi_{k+1} \Longrightarrow \\
a(\rho(t))< & a\left(\xi_{k+1}\right)=b\left(\xi_{k}\right), \text { and } \\
\left\|H_{k, 1}^{v}\right\|^{r} & =\left(\int_{s_{\sigma}}^{s_{\rho}} w^{q}(x) d x\right)^{\frac{r}{q}}\left(\int_{b\left(\xi_{k}\right)}^{b\left(s_{\sigma}\right)} k^{p^{\prime}}\left(s_{\sigma}, y\right) v^{p^{\prime}}(y) d y\right)^{\frac{r}{p^{\prime}}} \\
& \ll \int_{s_{\sigma}}^{s_{\rho}}\left(\int_{t}^{s_{\rho}} w^{q}(x) d x\right)^{\frac{r}{p}}\left(\int_{b\left(\xi_{k}\right)}^{b(t)} k^{p^{\prime}}(t, y) v^{p^{\prime}}(y) d y\right)^{\frac{r}{p^{\prime}}} w^{q}(t) d t \\
& <\int_{s_{\sigma}}^{s_{\rho}}\left(\int_{\delta^{+}(t)}^{\frac{r}{p}} w^{q}(x) d x\right)^{\frac{r}{p^{\prime}}}\left(\int_{\Delta(t)} k^{p^{\prime}}(t, y) v^{p^{\prime}}(y) d y\right)^{q}(t) d t \\
& \leq \int_{\Delta_{k}} \mathcal{B}_{\sigma}^{+}(t) d t
\end{aligned}
$$


on the strength of $a(t)<a\left(\xi_{k+1}\right)=b\left(\xi_{k}\right)$ and $a\left(s_{\rho}\right)<b\left(\xi_{k}\right)=\sigma\left(s_{\sigma}\right) \leq \sigma(t)$ $\Longrightarrow s_{\rho}<a^{-1}(\sigma(t))$. For $S_{k, 2}$ we use Corollary 2.1 with $c=s_{\sigma}$ and $d=s_{\rho}$ :

$$
\begin{aligned}
\left\|S_{k, 2}\right\|^{r}: & =\left\|S_{k, 2}\right\|_{L_{p}\left(b\left(s_{\sigma}\right), b\left(s_{\rho}\right)\right) \rightarrow L_{q}\left(s_{\sigma}, s_{\rho}\right)}^{r} \\
(4.29) \approx & B_{b, 0}^{r}+B_{b, 1}^{r} \\
= & \int_{b\left(s_{\sigma}\right)}^{b\left(s_{\rho}\right)}\left(\int_{b^{-1}(t)}^{s_{\rho}} k^{q}(x, t) w^{q}(x) d x\right)^{\frac{r}{q}}\left(\int_{b\left(s_{\sigma}\right)}^{t} v^{p^{\prime}}(y) d y\right)^{\frac{r}{q^{\prime}}} v^{p^{\prime}}(t) d t \\
& +\int_{s_{\sigma}}^{s_{\rho}}\left(\int_{t}^{s_{\rho}} w^{q}(x) d x\right)^{\frac{r}{p}}\left(\int_{b\left(s_{\sigma}\right)}^{b(t)} k^{p^{\prime}}(t, y) v^{p^{\prime}}(y) d y\right)^{p^{q}}(t) d t .
\end{aligned}
$$

Since $a\left(s_{\rho}\right)<b\left(s_{\sigma}\right) \leq t \leq b\left(s_{\rho}\right)=\rho^{-1}\left(\xi_{k+1}\right)$ we have in $B_{b, 0}$ that $s_{\rho}<a^{-1}(t)$ and $\rho(t) \leq \xi_{k+1} \Longrightarrow a(\rho(t)) \leq a\left(\xi_{k+1}\right)<b\left(s_{\sigma}\right)$. Therefore,

$$
\begin{aligned}
B_{b, 0}^{r} & \ll \int_{b\left(s_{\sigma}\right)}^{b\left(s_{\rho}\right)}\left(\int_{\Theta(t)} k^{q}(x, t) w^{q}(x) d x\right)^{\frac{r}{q}}\left(\int_{\vartheta^{-}(t)} v^{p^{\prime}}(y) d y\right)^{\frac{r}{q^{\prime}}} v^{p^{\prime}}(t) d \\
(4.30) & \leq \int_{\delta_{k+1}} \mathcal{B}_{\rho}^{-}(t) d t .
\end{aligned}
$$

In $B_{b, 1}$ in view of $a\left(s_{\rho}\right)<b\left(\xi_{k}\right)=\sigma\left(s_{\sigma}\right)<\sigma(t) \Longrightarrow s_{\rho}<a^{-1}(\sigma(t))$ and $a(t)<b\left(s_{\sigma}\right)$ we have

$$
\begin{aligned}
B_{b, 1}^{r} & \ll \int_{s_{\sigma}}^{s_{\rho}}\left(\int_{\delta^{+}(t)} w^{q}(x) d x\right)^{\frac{r}{p}}\left(\int_{\Delta(t)} k^{p^{\prime}}(t, y) v^{p^{\prime}}(y) d y\right)^{\frac{r}{p^{\prime}}} w^{q}(t) d t \\
& \leq \int_{\Delta_{k}} \mathcal{B}_{\sigma}^{+}(t) d t .
\end{aligned}
$$

Analogously to the case (i) it holds that

$$
\left\|H_{k, 2}\right\|:=\left\|H_{k, 2}\right\|_{L_{p}\left(b\left(\xi_{k}\right), b\left(s_{\rho}\right)\right) \rightarrow L_{q}\left(s_{\rho}, \xi_{k+1}\right)} \ll \int_{\delta_{k+1}} \mathcal{B}_{\rho}^{-}(t) d t+\int_{\Delta_{k}} \mathcal{B}_{\sigma}^{+}(t) d t
$$

and

$$
\left\|S_{k, 3}\right\|^{r}:=\left\|S_{k, 3}\right\|_{L_{p}\left(b\left(s_{\rho}\right), b\left(\xi_{k+1}\right)\right) \rightarrow L_{q}\left(s_{\rho}, \xi_{k+1}\right)}^{r} \ll \int_{\delta_{k+1}} \mathcal{B}_{\rho}^{+}(t) d t+\int_{\Delta_{k}} \mathcal{B}_{\sigma}^{+}(t) d t .
$$


Now from (4.25) - (4.33) we have the estimate (4.24) for the case (iii) too. The case (ii) is clear from either (i) or (iii). Now we obtain from (4.5) by Lemma 2.1

$$
\|\mathcal{S}\| \approx\left(\sum_{k}\left\|S_{k}\right\|_{L_{p}\left(\delta_{k+1}\right) \rightarrow L_{q}\left(\Delta_{k}\right)}^{r}\right)^{\frac{1}{r}} \ll \mathcal{B}_{\rho}+\mathcal{B}_{\sigma}
$$

To estimate the norm of the operator $T_{k}$ we decompose it by (1.3), (4.6) into the sum

$$
\begin{aligned}
T_{k} f(x) \approx & w(x) k\left(x, b\left(\xi_{k}\right)\right) \int_{a(x)}^{b\left(\xi_{k}\right)} f(y) v(y) d y \\
& +w(x) \int_{a(x)}^{b\left(\xi_{k}\right)} k\left(\xi_{k}, y\right) f(y) v(y) d y=: T_{k}^{w} f(x)+T_{k}^{v} f(x) .
\end{aligned}
$$

Then we find two points $t_{\rho}:=a^{-1}\left(\rho^{-1}\left(\xi_{k}\right)\right), t_{\sigma}:=\sigma^{-1}\left(b\left(\xi_{k}\right)\right)$ and make two more decompositions

$T_{k}^{w} f(x)=T_{k}^{w}\left(f \chi_{\left[a\left(\xi_{k}\right), \rho^{-1}\left(\xi_{k}\right)\right]}\right)(x)+T_{k}^{w}\left(f \chi_{\left[\rho^{-1}\left(\xi_{k}\right), b\left(\xi_{k}\right)\right]}\right)(x)=: T_{k, 1}^{w} f(x)+T_{k, 2}^{w} f(x)$,

$$
T_{k}^{v} f(x)=T_{k}^{v} f(x) \chi_{\left[\xi_{k}, t_{\sigma}\right]}(x)+T_{k}^{v} f(x) \chi_{\left[t_{\sigma}, \xi_{k+1}\right]}(x)=: T_{k, 1}^{v} f(x)+T_{k, 2}^{v} f(x)
$$

(see Figure 1 and Figure 2 respectively). By duality and (2.15) it follows from Lemma 2.4 with $c=a\left(\xi_{k}\right), d=\rho^{-1}\left(\xi_{k}\right), a=\xi_{k}, b(x) \rightarrow a^{-1}(y)$, $v(y) \rightarrow w(x) k\left(x, b\left(\xi_{k}\right)\right), w(x) \rightarrow v(y)$ and $q=p^{\prime}, p=q^{\prime}$ that

$$
\begin{aligned}
\left\|T_{k, 1}^{w}\right\|^{r}:= & \left\|T_{k, 1}^{w}\right\|_{L_{p}\left(a\left(\xi_{k}\right), \rho^{-1}\left(\xi_{k}\right)\right) \rightarrow L_{q}\left(\xi_{k}, t_{\rho}\right)}^{r} \approx \\
\approx & \int_{a\left(\xi_{k}\right)}^{\rho^{-1}\left(\xi_{k}\right)}\left(\int_{a\left(\xi_{k}\right)}^{t}\left[\int_{\xi_{k}}^{a^{-1}(y)} w^{q}(x) k^{q}\left(x, b\left(\xi_{k}\right)\right) d x\right]^{p^{\prime}} v^{p^{\prime}}(y) d y\right)^{\frac{r}{q^{\prime}}} \\
& \times\left(\int_{\xi_{k}}^{a^{-1}(t)} w^{q}(x) k^{q}\left(x, b\left(\xi_{k}\right)\right) d x\right)^{p^{\prime}-\frac{r}{q^{\prime}}} v^{p^{\prime}}(t) d t .
\end{aligned}
$$

Since $\xi_{k} \leq x$ and $a(x) \leq y \leq t \leq \rho^{-1}\left(\xi_{k}\right)<b\left(\xi_{k}\right)$ then $k\left(x, b\left(\xi_{k}\right)\right) \ll k(x, t)$ and, therefore,

$$
\left\|T_{k, 1}^{w}\right\|^{r} \ll \int_{a\left(\xi_{k}\right)}^{\rho^{-1}\left(\xi_{k}\right)}\left(\int_{a\left(\xi_{k}\right)}^{t} v^{p^{\prime}}(y) d y\right)^{\frac{r}{q^{\prime}}}\left(\int_{\xi_{k}}^{a^{-1}(t)} k^{q}(x, t) w^{q}(x) d x\right)^{\frac{r}{q}} v^{p^{\prime}}(t) d t
$$




$$
\begin{aligned}
& <\int_{a\left(\xi_{k}\right)}^{\rho^{-1}\left(\xi_{k}\right)}\left(\int_{\Theta(t)} k^{q}(x, t) w^{q}(x) d x\right)^{\frac{r}{q}}\left(\int_{\vartheta^{-}(t)} v^{p^{\prime}}(y) d y\right)^{\frac{r}{q^{\prime}}} v^{p^{\prime}}(t) d t \\
(4.38) & \leq \int_{\delta_{k}} \mathcal{B}_{\rho}^{-}(t) d t
\end{aligned}
$$

on the strength of $t<b\left(\xi_{k}\right) \Longrightarrow b^{-1}(t)<\xi_{k}$ and $t \leq \rho^{-1}\left(\xi_{k}\right) \Longrightarrow$ $a(\rho(t)) \leq a\left(\xi_{k}\right)$. Further, again by duality and (2.14), we obtain from Lemma 2.4 with $c=\rho^{-1}\left(\xi_{k}\right), d=b\left(\xi_{k}\right), a=\xi_{k}, b(x) \rightarrow a^{-1}(y), v(y) \rightarrow$ $w(x) k\left(x, b\left(\xi_{k}\right)\right), w(x) \rightarrow v(y), q=p^{\prime}, p=q^{\prime}$ provided $a^{-1}\left(\rho^{-1}\left(\xi_{k}\right)\right)=t_{\rho}$ that

$$
\begin{aligned}
& \left\|T_{k, 2}^{w}\right\|^{r}:=\left\|T_{k, 2}^{w}\right\|_{L_{p}\left(\rho^{-1}\left(\xi_{k}\right), b\left(\xi_{k}\right)\right) \rightarrow L_{q}\left(\xi_{k}, \xi_{k+1}\right)}^{r} \approx \\
& \approx \int_{\rho^{-1}\left(\xi_{k}\right)}^{b\left(\xi_{k}\right)}\left(\int_{\xi_{k}}^{a^{-1}(t)} w^{q}(x) k^{q}\left(x, b\left(\xi_{k}\right)\right) d x\right)^{\frac{r}{q}}\left(\int_{t}^{b\left(\xi_{k}\right)} v^{p^{\prime}}(y) d y\right)^{\frac{r}{q^{\prime}}} v^{p^{\prime}}(t) d t .
\end{aligned}
$$

Since $\xi_{k} \leq x$ and $a(x) \leq t \leq b\left(\xi_{k}\right)$ then $k\left(x, b\left(\xi_{k}\right)\right) \ll k(x, t)$ and, therefore,

$$
\left\|T_{k, 2}^{w}\right\|^{r} \ll \int_{\rho^{-1}\left(\xi_{k}\right)}^{b\left(\xi_{k}\right)}\left(\int_{\xi_{k}}^{a^{-1}(t)} k^{q}(x, t) w^{q}(x) d x\right)^{\frac{r}{q}}\left(\int_{t}^{b\left(\xi_{k}\right)} v^{p^{\prime}}(y) d y\right)^{\frac{r}{q^{\prime}}} v^{p^{\prime}}(t) d t
$$

$$
<\int_{\rho^{-1}\left(\xi_{k}\right)}^{b\left(\xi_{k}\right)}\left(\int_{\Theta(t)} k^{q}(x, t) w^{q}(x) d x\right)^{\frac{r}{q}}\left(\int_{\vartheta^{+}(t)} v^{p^{\prime}}(y) d y\right)^{\frac{r}{q^{\prime}}} v^{p^{\prime}}(t) d t \leq \int_{\delta_{k}} \mathcal{B}_{\rho}^{+}(t) d t
$$

on the strength of $t \leq b\left(\xi_{k}\right) \Longrightarrow b^{-1}(t) \leq \xi_{k}$ and $t \geq \rho^{-1}\left(\xi_{k}\right) \Longrightarrow$ $b\left(\xi_{k}\right) \leq b(\rho(t))$. Now we have by (2.16) from Lemma 2.5 and (1.3) with $\xi_{k} \leq t, a(t) \leq y \leq b\left(\xi_{k}\right)$

$$
\begin{aligned}
\left\|T_{k, 1}^{v}\right\|^{r} & :=\left\|T_{k, 1}^{v}\right\|_{L_{p}\left(a\left(\xi_{k}\right), b\left(\xi_{k}\right)\right) \rightarrow L_{q}\left(\xi_{k}, t_{\sigma}\right)}^{r} \approx \\
& \approx \int_{\xi_{k}}^{t_{\sigma}}\left(\int_{\xi_{k}}^{t} w^{q}(x) d x\right)^{\frac{r}{p}}\left(\int_{a(t)}^{b\left(\xi_{k}\right)} k^{p^{\prime}}\left(\xi_{k}, y\right) v^{p^{\prime}}(y) d y\right)^{\frac{r}{p^{\prime}}} w^{q}(t) d t \\
& \stackrel{(1.3)}{\ll} \int_{\xi_{k}}^{t_{\sigma}}\left(\int_{\xi_{k}}^{t} w^{q}(x) d x\right)^{\frac{r}{p}}\left(\int_{a(t)}^{b\left(\xi_{k}\right)} k^{p^{\prime}}(t, y) v^{p^{\prime}}(y) d y\right)^{\frac{r}{p^{\prime}}} w^{q}(t) d t
\end{aligned}
$$




$$
\begin{aligned}
& <\int_{\xi_{k}}^{t_{\sigma}}\left(\int_{\delta^{-}(t)} w^{q}(x) d x\right)^{\frac{r}{p}}\left(\int_{\Delta(t)} k^{p^{\prime}}(t, y) v^{p^{\prime}}(y) d y\right)^{\frac{r}{p^{\prime}}} w^{q}(t) d t \\
(4.40) & \leq \int_{\Delta_{k}} \mathcal{B}_{\sigma}^{-}(t) d t
\end{aligned}
$$

since $\sigma(t) \leq b\left(\xi_{k}\right) \Longrightarrow b^{-1}(\sigma(t)) \leq \xi_{k}$ and $b\left(\xi_{k}\right) \leq b(t)$. Finally, by (2.17), from Lemma 2.5 and (1.3) with $\xi_{k}<t_{\sigma} \leq t, a(t) \leq a(x) \leq y \leq b\left(\xi_{k}\right)$ we obtain

$$
\begin{aligned}
\left\|T_{k, 2}^{v}\right\|^{r}:= & \left\|T_{k, 2}^{v}\right\|_{L_{p}\left(a\left(t_{\sigma}\right), b\left(\xi_{k}\right)\right) \rightarrow L_{q}\left(t_{\sigma}, \xi_{k+1}\right)}^{r} \approx \\
\approx & \int_{t_{\sigma}}^{\xi_{k+1}}\left(\int_{t}^{\xi_{k+1}}\left[\int_{a(x)}^{b\left(\xi_{k}\right)} k^{p^{\prime}}\left(\xi_{k}, y\right) v^{p^{\prime}}(y) d y\right]^{q} w^{q}(x) d x\right)^{\frac{r}{p}} \\
& \times\left(\int_{a(t)}^{b\left(\xi_{k}\right)} k^{p^{\prime}}\left(\xi_{k}, y\right) v^{p^{\prime}}(y) d y\right)^{q-\frac{r}{p}} w^{q}(t) d t \\
\stackrel{(1.3)}{\ll} & \int_{t_{\sigma}}^{\xi_{k+1}}\left(\int_{t}^{\xi_{k+1}} w^{q}(x) d x\right)^{\frac{r}{p}}\left(\int_{a(t)}^{b\left(\xi_{k}\right)} k^{p^{\prime}}(t, y) v^{p^{\prime}}(y) d y\right)^{\frac{r}{p^{\prime}}} w^{q}(t) d t \\
< & \int_{t_{\sigma}}^{\xi_{k+1}}\left(\int_{\delta^{+}(t)} w^{q}(x) d x\right)^{\frac{r}{p}}\left(\int_{\Delta(t)} k^{p^{\prime}}(t, y) v^{p^{\prime}}(y) d y\right)^{\frac{r}{p^{\prime}}} w^{q}(t) d t \\
(4.41) \leq & \int_{\Delta_{k}} \mathcal{B}_{\sigma}^{+}(t) d t
\end{aligned}
$$

since $a\left(\xi_{k+1}\right)=b\left(\xi_{k}\right) \leq \sigma(t) \Longrightarrow \xi_{k+1} \leq a^{-1}(\sigma(t))$ and $b\left(\xi_{k}\right)<b(t)$. Now from $(4.35)$ - (4.41) we have

$$
\left\|T_{k}\right\|_{L_{p}\left(\delta_{k}\right) \rightarrow L_{q}\left(\Delta_{k}\right)}^{r} \ll \int_{\delta_{k}} \mathcal{B}_{\rho}(t) d t+\int_{\Delta_{k}} \mathcal{B}_{\sigma}(t) d t .
$$

Therefore, from (4.5) by Lemma 2.1

$$
\|\mathcal{T}\| \approx\left(\sum_{k}\left\|T_{k}\right\|_{L_{p}\left(\delta_{k}\right) \rightarrow L_{q}\left(\Delta_{k}\right)}^{r}\right)^{\frac{1}{r}} \ll \mathcal{B}_{\rho}+\mathcal{B}_{\sigma} .
$$

Thus and from (4.34) the upper estimate in (3.4) follows in view of (4.4).

The lower estimate. Suppose that the inequality

$$
\|\mathcal{K} f\|_{q} \leq\|\mathcal{K}\|\|f\|_{p}
$$


holds. To prove

$$
\|\mathcal{K}\|_{L_{p} \rightarrow L_{q}} \gg \mathcal{B}_{\rho}^{-}
$$

we take a point sequence $\left\{\xi_{k}\right\}_{k \in \mathbb{Z}} \subset(0, \infty)$ such that

$$
\xi_{0}=1, \quad \xi_{k}=\left(a^{-1} \circ b\right)^{k}(1), \quad k \in \mathbb{Z},
$$

and denote

$$
W_{\rho}(t)=\int_{b^{-1}(t)}^{a^{-1}(t)} k^{q}(x, t) w^{q}(x) d x, \quad V_{\rho}^{-}(t)=\int_{a(\rho(t))}^{t} v^{p^{\prime}}(y) d y .
$$

Note that $\left[W_{\rho}(t)\right]^{r / p q}\left[V_{\rho}^{-}(t)\right]^{r / p q^{\prime}}[v(t)]^{p^{\prime}-1}=\mathcal{B}_{\rho}^{-}(t)^{1 / p}$. If we put

$$
f_{\rho}(t):=\left[W_{\rho}(t)\right]^{r / p q}\left[V_{\rho}^{-}(t)\right]^{r / p q^{\prime}}[v(t)]^{p^{\prime}-1},
$$

then $\left\|f_{\rho}\right\|_{p}=\left(\mathcal{B}_{\rho}^{-}\right)^{r / p}$. Thus, since $\sqcup_{k}\left[\xi_{k}, \xi_{k+1}\right)=(0, \infty)$ and $(4.43)$ holds we have

$$
\begin{aligned}
\|\mathcal{K}\|\left(\mathcal{B}_{\rho}^{-}\right)^{r / p} \stackrel{(4.43)}{\geq}\left\|\mathcal{K} f_{\rho}\right\|_{q}=\left(\sum_{k} \int_{\xi_{k}}^{\xi_{k+1}}\left(\mathcal{K} f_{\rho}\right)^{q}(x) d x\right)^{\frac{1}{q}} \\
\quad \geq 2^{-1 / q}\left(\sum_{k} \int_{b^{-1}\left(\rho^{-1}\left(\xi_{k}\right)\right)}^{\xi_{k+1}}\left(\mathcal{K} f_{\rho}\right)^{q}(x) d x\right)^{\frac{1}{q}} .
\end{aligned}
$$

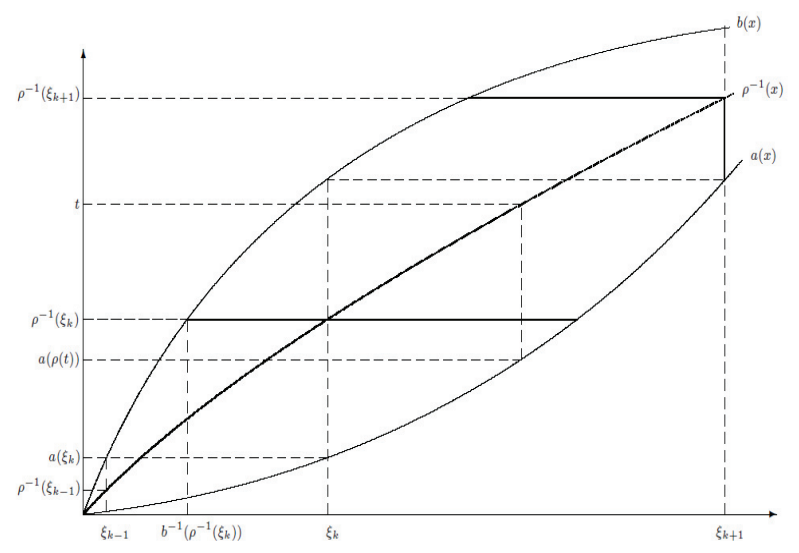

Figure 3. Necessity of $\mathcal{B}_{\rho}^{-}$ 
Using the explicit form of the operator $\mathcal{K}$ we find that

$$
\begin{aligned}
& \int_{b^{-1}\left(\rho^{-1}\left(\xi_{k}\right)\right)}^{\xi_{k+1}}\left(\mathcal{K} f_{\rho}\right)^{q}(x) d x \\
& =\int_{b^{-1}\left(\rho^{-1}\left(\xi_{k}\right)\right)}^{\xi_{k+1}} w^{q}(x)\left(\int_{a(x)}^{b(x)} k(x, y) f_{\rho}(y) v(y) d y\right)^{q} d x \\
& =\int_{b^{-1}\left(\rho^{-1}\left(\xi_{k}\right)\right)}^{\xi_{k+1}} w^{q}(x)\left(\int_{a(x)}^{b(x)} k(x, t) f_{\rho}(t) v(t) d t\right) \times \\
& \times\left(\int_{a(x)}^{b(x)} k(x, y) f_{\rho}(y) v(y) d y\right)^{q-1} d x \\
& \geq \int_{\rho^{-1}\left(\xi_{k}\right)}^{\rho^{-1}\left(\xi_{k+1}\right)} f_{\rho}(t) v(t)\left(\int_{b^{-1}(t)}^{\min \left\{a^{-1}(t), \xi_{k+1}\right\}} k(x, t) w^{q}(x) \times\right. \\
& \left.\times\left[\int_{a(x)}^{b(x)} k(x, y) f_{\rho}(y) v(y) d y\right]^{q-1} d x\right) d t \\
& \geq \int_{\rho^{-1}\left(\xi_{k}\right)}^{\rho^{-1}\left(\xi_{k+1}\right)} f_{\rho}(t) v(t)\left(\int_{b^{-1}(t)}^{\rho(t)} k(x, t) w^{q}(x) \times\right. \\
& \left.\times\left[\int_{a(x)}^{t} k(x, y) f_{\rho}(y) v(y) d y\right]^{q-1} d x\right) d t \\
& \text { [applying (1.3) with } z=b^{-1}(t) \\
& \gg \int_{\rho^{-1}\left(\xi_{k}\right)}^{\rho^{-1}\left(\xi_{k+1}\right)} f_{\rho}(t) v(t)\left(\int_{b^{-1}(t)}^{\rho(t)} k^{q}(x, t) w^{q}(x) \times\right. \\
& \left.\times\left[\int_{a(x)}^{t} f_{\rho}(y) v(y) d y\right]^{q-1} d x\right) d t \\
& \geq \int_{\rho^{-1}\left(\xi_{k}\right)}^{\rho^{-1}\left(\xi_{k+1}\right)} f_{\rho}(t) v(t)\left(\int_{b^{-1}(t)}^{\rho(t)} k^{q}(x, t) w^{q}(x) d x\right) \times \\
& \times\left(\int_{a(\rho(t))}^{t} f_{\rho}(y) v(y) d y\right)^{q-1} d t \\
& =\int_{\rho^{-1}\left(\xi_{k}\right)}^{\rho^{-1}\left(\xi_{k+1}\right)}\left[W_{\rho}(t)\right]^{r / p q}\left[V_{\rho}^{-}(t)\right]^{r / p q^{\prime}}\left(\int_{b^{-1}(t)}^{\rho(t)} k^{q}(x, t) w^{q}(x) d x\right) \\
& \times\left(\int_{a(\rho(t))}^{t}\left[W_{\rho}(y)\right]^{r / p q}\left[V_{\rho}^{-}(y)\right]^{r / p q^{\prime}} v^{p^{\prime}}(y) d y\right)^{q-1} v^{p^{\prime}}(t) d t
\end{aligned}
$$




$$
\begin{aligned}
& \stackrel{(3.1)}{\gg} \int_{\rho^{-1}\left(\xi_{k}\right)}^{\rho^{-1}\left(\xi_{k+1}\right)}\left[V_{\rho}^{-}(t)\right]^{r / p q^{\prime}}\left[W_{\rho}(t)\right]^{r / p q+1} \\
& \quad \times\left(\int_{a(\rho(t))}^{t}\left[\int_{b^{-1}(t)}^{\rho(t)} k^{q}(z, y) w^{q}(z) d z\right]^{\frac{r}{p q}}\left[V_{\rho}^{-}(y)\right]^{r / p q^{\prime}} v^{p^{\prime}}(y) d y\right)^{q-1} v^{p^{\prime}}(t) d t .
\end{aligned}
$$

It follows from (1.3) that $k(z, y) \gg k(z, t)$ provided $a(z) \leq a(\rho(t)) \leq y \leq$ $t=b(\tau), \tau=b^{-1}(t) \leq z$. Therefore, in view of (3.1)

$$
\begin{aligned}
\int_{b^{-1}\left(\rho^{-1}\left(\xi_{k}\right)\right)}^{\xi_{k+1}}\left(\mathcal{K} f_{\rho}\right)^{q}(x) d x & \gg \int_{\rho^{-1}\left(\xi_{k}\right)}^{\rho^{-1}\left(\xi_{k+1}\right)}\left[V_{\rho}^{-}(t)\right]^{r / p q^{\prime}}\left[W_{\rho}(t)\right]^{r / q} \\
& \left.\times \int_{a(\rho(t))}^{t}\left[\int_{a(\rho(t))}^{y} v^{p^{\prime}}(z) d z\right]^{r / p q^{\prime}} v^{p^{\prime}}(y) d y\right)^{q-1} v^{p^{\prime}}(t) d t \\
& \approx \int_{\rho^{-1}\left(\xi_{k}\right)}^{\rho^{-1}\left(\xi_{k+1}\right)} \mathcal{B}_{\rho}^{-}(t) d t
\end{aligned}
$$

Since $\sqcup_{k}\left[\rho^{-1}\left(\xi_{k}\right), \rho^{-1}\left(\xi_{k+1}\right)\right)=(0, \infty)$, it yields

$$
\left(\sum_{k} \int_{b^{-1}\left(\rho^{-1}\left(\xi_{k}\right)\right)}^{\xi_{k+1}}\left(\mathcal{K} f_{\rho}\right)^{q}(x) d x\right)^{\frac{1}{q}} \gg\left(\mathcal{B}_{\rho}^{-}\right)^{r / q}
$$

Thus, by (4.46) we obtain (4.44).

To prove

$$
\|\mathcal{K}\|_{L_{p} \rightarrow L_{q}} \gg \mathcal{B}_{\sigma}^{+}
$$

we use the dual to (4.43) inequality

$$
\left(\int_{0}^{\infty}\left[\int_{b^{-1}(y)}^{a^{-1}(y)} k(x, y) g(x) w(x) d x\right]^{p^{\prime}} v^{p^{\prime}}(y) d y\right)^{\frac{1}{p^{\prime}}} \leq\|\overline{\mathcal{K}}\|\left(\int_{0}^{\infty} g^{q^{\prime}}(x) d x\right)^{\frac{1}{q^{\prime}}}
$$

Note that the kernel $k(x, y)$ is satisfying a condition following from (1.3) : (4.49) $D^{-1} k(x, y) \leq k(x, z)+k\left(b^{-1}(z), y\right) \leq D k(x, y), y \leq z, \quad b^{-1}(z) \leq x \leq a^{-1}(y)$. 
Break the semiaxis $(0, \infty)$ by the point sequence $(4.45)$ and put

$$
W_{\sigma}^{+}(t)=\int_{t}^{a^{-1}(\sigma(t))} w^{q}(x) d x, \quad V_{\sigma}(t)=\int_{a(t)}^{b(t)} k^{p^{\prime}}(t, y) v^{p^{\prime}}(y) d y .
$$

Note that $\left[W_{\sigma}^{+}(t)\right]^{r / p q^{\prime}}\left[V_{\sigma}(t)\right]^{r / p^{\prime} q^{\prime}}[w(t)]^{q-1}=\mathcal{B}_{\sigma}^{+}(t)^{1 / q^{\prime}}$. If we take

$$
g_{\sigma}(t)=\left[W_{\sigma}^{+}(t)\right]^{r / p q^{\prime}}\left[V_{\sigma}(t)\right]^{r / p^{\prime} q^{\prime}}[w(t)]^{q-1},
$$

then $\left(\int_{0}^{\infty} g_{\sigma}^{q^{\prime}}(x) d x\right)^{\frac{1}{q^{\prime}}}=\left(\mathcal{B}_{\sigma}^{+}\right)^{r / q^{\prime}}$. Thus, by $\sqcup_{k}\left[\sigma\left(\xi_{k}\right), \sigma\left(\xi_{k+1}\right)\right)=(0, \infty)$ and (4.48), we have

$$
\begin{aligned}
\|\overline{\mathcal{K}}\|\left(\mathcal{B}_{\sigma}^{+}\right)^{r / q^{\prime}} \stackrel{(4.48)}{\geq}\left\|\overline{\mathcal{K}} g_{\sigma}\right\|_{p^{\prime}}=\left(\sum_{k} \int_{\sigma\left(\xi_{k}\right)}^{\sigma\left(\xi_{k+1}\right)}\left(\overline{\mathcal{K}} g_{\sigma}\right)^{p^{\prime}}(y) d y\right)^{\frac{1}{p^{\prime}}} \\
\quad \geq 2^{-1 / p^{\prime}}\left(\sum_{k} \int_{\sigma\left(\xi_{k}\right)}^{b\left(\xi_{k+1}\right)}\left(\overline{\mathcal{K}} g_{\sigma}\right)^{p^{\prime}}(y) d y\right)^{\frac{1}{p^{\prime}}}
\end{aligned}
$$

where $\overline{\mathcal{K}} g(y):=v(y) \int_{b^{-1}(y)}^{a^{-1}(y)} k(x, y) g(x) w(x) d x$. We find that

$$
\begin{aligned}
& \int_{\sigma\left(\xi_{k}\right)}^{b\left(\xi_{k+1}\right)}\left(\overline{\mathcal{K}} g_{\sigma}\right)^{p^{\prime}}(y) d y=\int_{\sigma\left(\xi_{k}\right)}^{b\left(\xi_{k+1}\right)} v^{p^{\prime}}(y)\left(\int_{b^{-1}(y)}^{a^{-1}(y)} k(x, y) g_{\sigma}(x) w(x) d x\right)^{p^{\prime}} d y \\
& =\int_{\sigma\left(\xi_{k}\right)}^{b\left(\xi_{k+1}\right)} v^{p^{\prime}}(y)\left(\int_{b^{-1}(y)}^{a^{-1}(y)} k(t, y) g_{\sigma}(t) w(t) d t\right) \times \\
& \quad \times\left(\int_{b^{-1}(y)}^{a^{-1}(y)} k(x, y) g_{\sigma}(x) w(x) d x\right)^{p^{\prime}-1} d x \\
& \geq \int_{\xi_{k}}^{\xi_{k+1}} g_{\sigma}(t) w(t)\left(\int_{\max \left\{a(t), \sigma\left(\xi_{k}\right)\right\}}^{b(t)} k(t, y) v^{p^{\prime}}(y) \times\right. \\
& \left.\quad \times\left[\int_{b^{-1}(y)}^{a^{-1}(y)} k(x, y) g_{\sigma}(x) w(x) d x\right]^{p^{\prime}-1} d y\right) d t \\
& \geq \int_{\xi_{k}}^{\xi_{k+1}} g_{\sigma}(t) w(t)\left(\int_{\sigma(t)}^{b(t)} k(t, y) v^{p^{\prime}}(y) \times\right. \\
& \left.\quad \times\left[\int_{t}^{a^{-1}(y)} k(x, y) g_{\sigma}(x) w(x) d x\right]^{p^{\prime}-1} d y\right) d t
\end{aligned}
$$


[applying (4.49) with $z=b(t)$ ]

$$
\begin{aligned}
& \gg \int_{\xi_{k}}^{\xi_{k+1}} g_{\sigma}(t) w(t)\left(\int_{\sigma(t)}^{b(t)} k^{p^{\prime}}(t, y) v^{p^{\prime}}(y)\left[\int_{t}^{a^{-1}(y)} g_{\sigma}(x) w(x) d x\right]^{p^{\prime}-1} d y\right) d t \\
& \geq \int_{\xi_{k}}^{\xi_{k+1}} g_{\sigma}(t) w(t)\left(\int_{\sigma(t)}^{b(t)} k^{p^{\prime}}(t, y) v^{p^{\prime}}(y) d y\right)\left(\int_{t}^{a^{-1}(\sigma(t))} g_{\sigma}(x) w(x) d x\right)^{p^{\prime}-1} d t \\
& =\int_{\xi_{k}}^{\xi_{k+1}}\left[W_{\sigma}^{+}(t)\right]^{r / p q^{\prime}}\left[V_{\sigma}(t)\right]^{r / p^{\prime} q^{\prime}}\left(\int_{\sigma(t)}^{b(t)} k^{p^{\prime}}(t, y) v^{p^{\prime}}(y) d y\right) \\
& \times\left(\int_{t}^{a^{-1}(\sigma(t))}\left[W_{\sigma}^{+}(x)\right]^{r / p q^{\prime}}\left[V_{\sigma}(x)\right]^{r / p^{\prime} q^{\prime}} w^{q}(x) d x\right)^{p^{\prime}-1} w^{q}(t) d t \\
& \stackrel{(3.2)}{\gg} \int_{\xi_{k}}^{\xi_{k+1}}\left[W_{\sigma}^{+}(t)\right]^{r / p q^{\prime}}\left[V_{\sigma}(t)\right]^{r / p^{\prime} q^{\prime}+1} \\
& \times\left(\int_{t}^{a^{-1}(\sigma(t))}\left[\int_{\sigma(t)}^{b(t)} k^{p^{\prime}}(x, z) v^{p^{\prime}}(z) d z\right]^{\frac{r}{p^{\prime} q^{\prime}}}\left[W_{\sigma}^{+}(x)\right]^{r / p q^{\prime}} w^{q}(x) d x\right)^{p^{\prime}-1} w^{q}(t) d t .
\end{aligned}
$$

It follows from (4.49) that $k(x, z) \gg k(t, z)$ because $b^{-1}(\tau)=t \leq x \leq$ $a^{-1}(\sigma(t)) \leq a^{-1}(z)$ and $z \leq \tau=b(t)$. Therefore, by $(3.2)$

$$
\begin{aligned}
& \int_{\sigma\left(\xi_{k}\right)}^{b\left(\xi_{k+1}\right)}\left(\overline{\mathcal{K}}_{\sigma}\right)^{p^{\prime}}(y) d y \\
& \gg \int_{\xi_{k}}^{\xi_{k+1}}\left[W_{\sigma}^{+}(t)\right]^{r / p q^{\prime}}\left[V_{\sigma}(t)\right]^{r / p^{\prime}} \\
& \quad \times\left(\int_{t}^{a^{-1}(\sigma(t))}\left[\int_{x}^{a^{-1}(\sigma(t))} w^{q}(z) d z\right]^{r / p q^{\prime}} w^{q}(x) d x\right)^{p^{\prime}-1} w^{q}(t) d t \\
& \approx \int_{\xi_{k}}^{\xi_{k+1}} \mathcal{B}_{\sigma}^{+}(t) d t .
\end{aligned}
$$

Thus and from (4.50)

$$
\|\mathcal{K}\|_{L_{p} \rightarrow L_{q}}=\|\overline{\mathcal{K}}\|_{L_{q^{\prime}} \rightarrow L_{p^{\prime}}} \gg \mathcal{B}_{\sigma}^{+} .
$$

Combining (4.44) and (4.51) we obtain the lower estimate in (3.4). The assertion about compactness for $q<p$ is a direct corollary of the obtained boundedness criterion and Ando's theorem. 


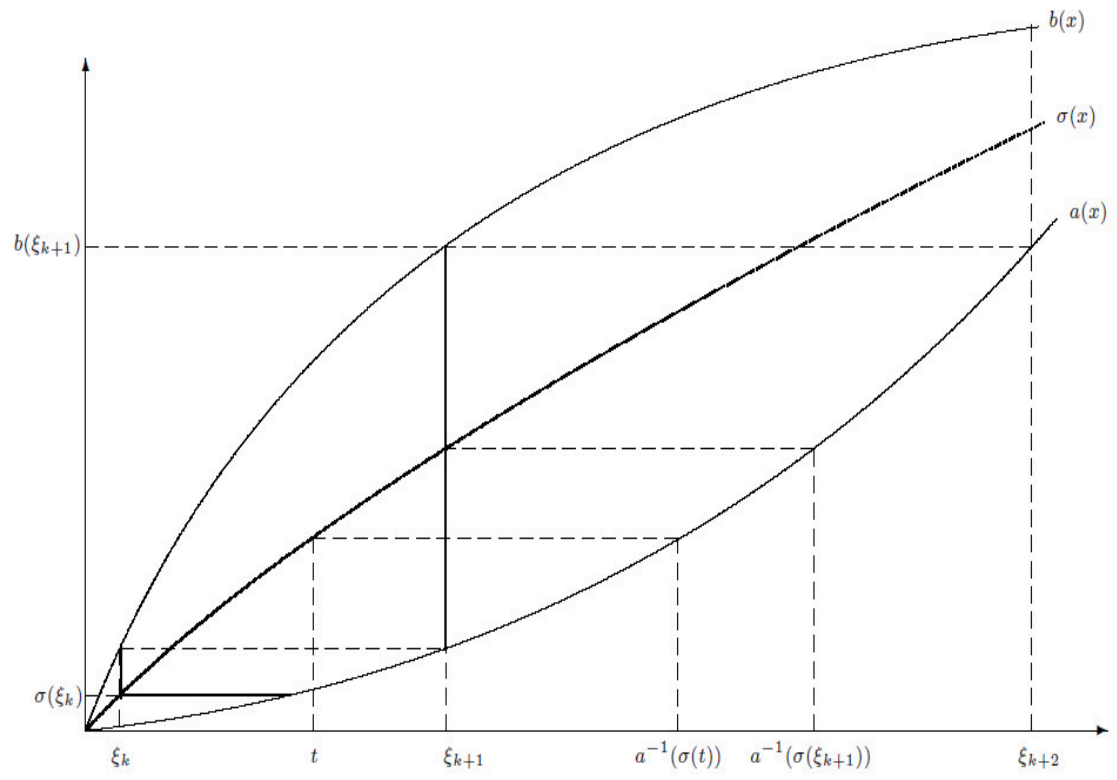

FIGURE 4. Necessity of $\mathcal{B}_{\sigma}^{+}$

The assertions of Theorem 3.2 can be proved analogously by using (1.8), Corollary 2.2, Lemma 2.3 and Lemmas 2.4, 2.5.

\section{Criterion cases}

Here we consider two cases when the results of Theorems 3.1 and 3.2 give criteria.

Theorem 5.1. Let the operator $\mathcal{K}$ be defined by (1.1) with $a(x), b(x)$ satisfying (1.2) and a continuous kernel $k(x, y)>0$. Suppose that the functions $\rho(x), \sigma(x)$ on $\mathbb{R}^{+}$are strictly increasing fairways from Definition 3.1.

(a) If $k(x, y) \in \mathcal{O}_{b}$ and

$$
\begin{array}{ll}
\int_{\vartheta^{-}(t)} v^{p^{\prime}}(y) d y \approx \int_{\vartheta(t)} v^{p^{\prime}}(y) d y, & t>0, \\
\int_{\delta^{+}(t)} w^{q}(x) d x \approx \int_{\delta(t)} w^{q}(x) d x, & t>0,
\end{array}
$$


then

$$
\|\mathcal{K}\|_{L_{p} \rightarrow L_{q}} \approx \begin{cases}\mathcal{A}_{\rho}+\mathcal{A}_{\sigma}, & 1<p \leq q<\infty \\ \mathcal{B}_{\rho}+\mathcal{B}_{\sigma}, & 1<q<p<\infty\end{cases}
$$

and $\mathcal{K}$ is compact if and only if $\mathcal{A}_{\rho}, \mathcal{A}_{\sigma}<\infty, \lim _{t \rightarrow 0} \mathcal{A}_{\rho}(t)=$ $\lim _{t \rightarrow 0} \mathcal{A}_{\sigma}(t)=0$ in the case $1<p \leq q<\infty$ and if and only if $\stackrel{t}{\mathcal{B}}_{\rho}, \mathcal{B}_{\sigma}<\infty$ for $\stackrel{t \rightarrow \infty}{1}<q<p<\infty$.

(b) If $k(x, y) \in \mathcal{O}_{a}$ and

$$
\begin{array}{ll}
\int_{\vartheta^{+}(t)} v^{p^{\prime}}(y) d y \approx \int_{\vartheta(t)} v^{p^{\prime}}(y) d y, & t>0, \\
\int_{\delta^{-}(t)} w^{q}(x) d x \approx \int_{\delta(t)} w^{q}(x) d x, & t>0,
\end{array}
$$

then the estimate (5.3) holds and $\mathcal{K}$ is compact if and only if $\mathcal{A}_{\rho}, \mathcal{A}_{\sigma}<\infty$, $\lim _{t \rightarrow 0} \mathcal{A}_{\rho}(t)=\lim _{t \rightarrow 0} \mathcal{A}_{\sigma}(t)=0$ in the case $1<p \leq q<\infty$ and if and only if $\mathcal{B}_{\rho}, \mathcal{B}_{\sigma}<\infty$ for $1<q<p<\infty$.

Theorem 5.2. Let the operator $\mathcal{K}$ be given by (1.1) with $a(x), b(x) \in$ (1.2) and a continuous kernel $0<k(x, y) \in \mathcal{O}_{a} \cap \mathcal{O}_{b}$. Suppose $\rho(x), \sigma(x)$ on $\mathbb{R}^{+}$are strictly increasing fairways satisfying Definition 3.1

(a) If $1<p \leq q<\infty$, then

$$
\alpha_{7}(p, q)\left[\mathcal{A}_{\rho}+\mathcal{A}_{\sigma}\right] \leq\|\mathcal{K}\|_{L_{p} \rightarrow L_{q}} \leq \alpha_{8}(p, q)\left[\mathcal{A}_{\rho}+\mathcal{A}_{\sigma}\right],
$$

and $\mathcal{K}$ is compact if and only if $\mathcal{A}_{\rho}, \mathcal{A}_{\sigma}<\infty, \lim _{t \rightarrow 0} \mathcal{A}_{\rho}(t)=$ $\lim _{t \rightarrow 0} \mathcal{A}_{\sigma}(t)=0$.

(b) If $1<q<p<\infty$ then

$$
\beta_{7}(p, q)\left[\mathcal{B}_{\rho}+\mathcal{B}_{\sigma}\right] \leq\|\mathcal{K}\|_{L_{p} \rightarrow L_{q}} \leq \beta_{8}(p, q)\left[\mathcal{B}_{\rho}+\mathcal{B}_{\sigma}\right],
$$

and $\mathcal{K}$ is compact if and only if $\mathcal{B}_{\rho}, \mathcal{B}_{\sigma}<\infty$.

Proofs of Theorems 5.1 and 5.2 easy follow from Theorems 3.1 and 3.2.

\section{Examples}

We conclude the paper by illustrating some of our results by examples. The first of them is about a criterion case from Theorem 5.1. 
Example 6.1. Let $p=q=2, v(y)=y^{-3 / 2}, w(x)=1, k(x, y)=$ $(y-a(x))^{1 / 2}, a(x)=x / 2, b(x)=2 x$, that is

$$
K_{1} f(x)=\int_{x / 2}^{2 x}(y-x / 2)^{1 / 2} f(y) y^{-3 / 2} d y .
$$

According to (3.1)

$$
L_{v}(x):=\int_{x / 2}^{\sigma(x)}(y-x / 2) y^{-3} d y=\int_{\sigma(x)}^{2 x}(y-x / 2) y^{-3} d y=R_{v}(x) .
$$

Integrating by parts we find

$$
\begin{aligned}
L_{v}(x) & =-\frac{1}{2} \int_{x / 2}^{\sigma(x)}(y-x / 2) d y^{-2} \\
& =-\frac{1}{2}(\sigma(x)-x / 2) \sigma^{-2}(x)-\frac{1}{2} \int_{x / 2}^{\sigma(x)} d y^{-1} \\
& =\frac{1}{x}-\sigma^{-1}(x)+\frac{x}{4} \sigma^{-2}(x)
\end{aligned}
$$

and

$$
R_{v}(x)=-\frac{1}{2} \int_{\sigma(x)}^{2 x}(y-x / 2) d y^{-2}=-\frac{5}{8 x}+\sigma^{-1}(x)-\frac{x}{4} \sigma^{-2}(x) .
$$

Thus, since $x / 2 \leq \sigma(x) \leq 2 x$

$$
\sigma(x)=\frac{2(4+\sqrt{3})}{13} x .
$$

Analogously, on the strength of (3.2)

$$
L_{w}(y):=\int_{y / 2}^{\rho(y)}(y-x / 2) d x=\int_{\rho(y)}^{2 y}(y-x / 2) d x=: R_{w}(y) .
$$

We have

$$
L_{w}(y)=\int_{y-\rho(y) / 2}^{y-y / 4} d z^{2}=\left(\frac{3}{4} y\right)^{2}-\left(y-\frac{\rho(y)}{2}\right)^{2}
$$

and

$$
R_{w}(y)=\int_{0}^{y-\rho(y) / 2} d z^{2}=\left(y-\frac{\rho(y)}{2}\right)^{2} .
$$

Therefore,

$$
\rho(y)=\frac{4 \sqrt{2}-3}{2 \sqrt{2}} y .
$$


The operator $K_{1}$ with $k(x, y)=(y-a(x))^{1 / 2} \in \mathcal{O}_{a}$ is bounded from $L_{2}$ to $L_{2}$. Indeed, since

$$
\begin{aligned}
A_{\rho}: & =\sup _{t>0}\left(\int_{t / 2}^{2 t}(t-x / 2) d x\right)^{\frac{1}{2}}\left(\int_{\frac{4 \sqrt{2}-3}{4 \sqrt{2}} t}^{\frac{4 \sqrt{2}-3}{\sqrt{2}} t} y^{-3} d y\right)^{\frac{1}{2}} \\
& =\sup _{t>0} \frac{1}{\sqrt{2}}\left(\int_{0}^{\frac{3}{4} t} d z^{2}\right)^{\frac{1}{2}}\left(\int_{\frac{4 \sqrt{2}-3}{\sqrt{2}} t}^{\frac{4 \sqrt{2}-3}{4 \sqrt{2}} t} d y^{-2}\right)^{\frac{1}{2}}=\frac{3 \sqrt{15}}{4(4 \sqrt{2}-3)}<\infty
\end{aligned}
$$

and

$$
\begin{aligned}
A_{\sigma}: & =\sup _{t>0}\left(\int_{\frac{4+\sqrt{3}}{13} t}^{\frac{4(4+\sqrt{3})}{13} t} d x\right)^{\frac{1}{2}}\left(\int_{t / 2}^{2 t}(y-t / 2) y^{-3} d y\right)^{\frac{1}{2}} \\
& =\sup _{t>0}\left(\frac{3(4+\sqrt{3})}{13} t\right)^{\frac{1}{2}}\left(\frac{15}{16 t}\right)^{\frac{1}{2}}=\frac{3}{4} \sqrt{\frac{5(4+\sqrt{3})}{13}}<\infty
\end{aligned}
$$

by (3.3) we have $\left\|K_{1}\right\|_{L_{2} \rightarrow L_{2}}<\infty$. Moreover, since

$$
\begin{gathered}
\int_{\vartheta^{+}(t)} v^{p^{\prime}}(y) d y=\int_{t}^{\frac{4 \sqrt{2}-3}{\sqrt{2}} t} y^{-3} d y=\frac{39-24 \sqrt{2}}{2(41-24 \sqrt{2})} t^{-2}, \\
\int_{\vartheta(t)} v^{p^{\prime}}(y) d y=\int_{\frac{4 \sqrt{2}-3}{4 \sqrt{2}} t}^{\frac{4 \sqrt{2}-3}{\sqrt{2}} t} y^{-3} d y=\frac{15}{41-24 \sqrt{2}} t^{-2}
\end{gathered}
$$

and

$$
\begin{gathered}
\int_{\delta^{-}(t)} w^{q}(x) d x=\int_{\frac{4+\sqrt{3}}{13} t}^{t} d x=\frac{9-\sqrt{3}}{13} t \\
\int_{\delta(t)} w^{q}(x) d x=\int_{\frac{4+\sqrt{3}}{13} t}^{\frac{4(4+\sqrt{3})}{13} t} d x=\frac{3(4+\sqrt{3})}{13} t,
\end{gathered}
$$

the requirements (5.4), (5.5) are satisfied and on the strength of Theorem 5.1 (b) the conditions $A_{\rho}<\infty, A_{\sigma}<\infty$ are also necessary for the boundedness of the operator $K_{1}: L_{2} \rightarrow L_{2}$.

Note that proofs of upper norm estimates in Theorems 3.1, 3.2 do not use fairway's integral properties (3.1), (3.2). Therefore, if we need to state boundedness of $\mathcal{K}$ only, it is correct and easier to take a strongly increasing fairway function $\phi(x)$ with the only property $a(x)<\phi(x)<b(x), x>0$, instead of $\sigma(x)$ and $\rho^{-1}(x)$ equipped by (3.1), (3.2). 
Example 6.2. Let $p=3, q=2, k(x, y)=\left|\int_{y}^{x} h(z) d z\right|^{\gamma}$ with $\gamma>0$, $v(y)=h(y)^{2 / 3}, w(x)=h(x)^{1 / 2}$, where $0 \leq h \in L_{l o c}\left(\mathbb{R}^{+}\right), a(x)=\ln \sqrt{x+1}$ and $b(x)=\exp 2 x-1$. That is

$$
\begin{aligned}
K_{2} f(x)= & h(x)^{1 / 2} \int_{\ln \sqrt{x+1}}^{\exp 2 x-1}\left|\int_{y}^{x} h(z) d z\right|^{\gamma} f(y) h(y)^{2 / 3} d y \\
= & h(x)^{1 / 2} \int_{\ln \sqrt{x+1}}^{x}\left(\int_{y}^{x} h(z) d z\right)^{\gamma} f(y) h(y)^{2 / 3} d y \\
& +h(x)^{1 / 2} \int_{x}^{\exp 2 x-1}\left(\int_{x}^{y} h(z) d z\right)^{\gamma} f(y) h(y)^{2 / 3} d y \\
=: & K_{2,1} f(x)+K_{2,2} f(x),
\end{aligned}
$$

$p^{\prime}=3 / 2, q^{\prime}=2$ and $r=6$. Note that the kernel $k_{2,1}(x, y):=\left(\int_{y}^{x} h(z) d z\right)^{\gamma}$ of the operator $K_{2,1}$ is from the class $\mathcal{O}_{b}$, while the kernel $k_{2,2}(x, y):=$ $\left(\int_{x}^{y} h(z) d z\right)^{\gamma}$ of $K_{2,2}$ is of the type $\mathcal{O}_{a}$.

To state boundedness of $K_{2,1}$ with $h(z)=\exp (-z)$ we shall use the line $\phi_{1}(x)=x / 2$, which is $a(x)=\ln \sqrt{x+1}<\phi_{1}(x)<x=: b_{1}(x)$, instead of $\rho^{-1}(x)$ and $\sigma(x)$. Since $b_{1}^{-1}(t)=t, a^{-1}(t)=\exp 2 t-1, \phi_{1}^{-1}(t)=2 t$ then

$$
\begin{aligned}
& B_{1, \rho=\phi^{-1}}^{6}:= \\
& :=\int_{0}^{\infty}\left(\int_{t}^{\exp 2 t-1}\left[\int_{t}^{x} h(z) d z\right]^{2 \gamma} h(x) d x\right)^{3}\left(\int_{\ln \sqrt{2 t+1}}^{2 t} h(y) d y\right)^{3} h(t) d t \\
& =\int_{0}^{\infty}\left(\int_{t}^{\exp 2 t-1}\left[\int_{t}^{x} h(z) d z\right]^{2 \gamma} d \int_{t}^{x} h(z) d z\right)^{3}\left(\int_{\ln \sqrt{2 t+1}}^{2 t} h(y) d y\right)^{3} h(t) d t \\
& =\frac{1}{(2 \gamma+1)^{3}} \int_{0}^{\infty}\left(\int_{t}^{\exp 2 t-1} h(z) d z\right)^{3(2 \gamma+1)}\left(\int_{\ln \sqrt{2 t+1}}^{2 t} h(y) d y\right)^{3} h(t) d t
\end{aligned}
$$

and

$$
\begin{aligned}
& B_{1, \sigma=\phi}^{6}:= \\
& :=\int_{0}^{\infty}\left(\int_{t / 2}^{\exp t-1} h(x) d x\right)^{2}\left(\int_{\ln \sqrt{t+1}}^{t}\left[\int_{y}^{t} h(z) d z\right]^{3 \gamma / 2} h(y) d y\right)^{4} h(t) d t \\
& \left.=\int_{0}^{\infty}\left(\int_{t / 2}^{\exp t-1} h(x) d x\right)^{2}\left(\int_{\ln \sqrt{t+1}}^{t}\left[\int_{y}^{t} h(z) d z\right]^{3 \gamma / 2} d-\int_{y}^{t} h(z) d z\right]\right)^{4} h(t) d t \\
& =\left(\frac{2}{3 \gamma+2}\right)^{4} \int_{0}^{\infty}\left(\int_{t / 2}^{\exp t-1} h(x) d x\right)^{t}\left(\int_{\ln \sqrt{t+1}}^{t} h(z) d z\right)^{6 \gamma+4} h(t) d t .
\end{aligned}
$$


Let $h(z)=\exp (-z)$, then

$$
\begin{aligned}
B_{1, \rho=\phi^{-1}}^{6}= & \frac{1}{(2 \gamma+1)^{3}} \int_{0}^{\infty}\left(\int_{t}^{\exp 2 t-1} \exp (-z) d z\right)^{3(2 \gamma+1)} \\
& \times\left(\int_{\ln \sqrt{2 t+1}}^{2 t} \exp (-y) d y\right)^{3} \exp (-t) d t \\
\leq & \frac{1}{(2 \gamma+1)^{3}} \int_{0}^{\infty} \exp (-3(2 \gamma+1) t) \frac{1}{(2 t+1)^{3 / 2}} \exp (-t) d t \\
= & \frac{1}{(2 \gamma+1)^{3}} \int_{0}^{\infty} \frac{\exp (-2(3 \gamma+2) t)}{(2 t+1)^{3 / 2}} d t \\
\leq & \frac{1}{(2 \gamma+1)^{3}} \int_{0}^{\infty} \exp (-2(3 \gamma+2) t) d t \\
= & \frac{1}{2(3 \gamma+2)(2 \gamma+1)^{3}}<\infty
\end{aligned}
$$

and

$$
\begin{aligned}
B_{1, \sigma=\phi}^{6}= & \left(\frac{2}{3 \gamma+2}\right)^{4} \int_{0}^{\infty}\left(\int_{t / 2}^{\exp t-1} \exp (-x) d x\right)^{2} \\
& \times\left(\int_{\ln \sqrt{t+1}}^{t} \exp (-z) d z\right)^{6 \gamma+4} \exp (-t) d t \\
\leq & \left(\frac{2}{3 \gamma+2}\right)^{4} \int_{0}^{\infty} \frac{\exp (-2 t)}{(t+1)^{3 \gamma+2}} d t \leq \frac{8}{(3 \gamma+2)^{4}}<\infty .
\end{aligned}
$$

Thus, by Theorem 3.1 the operator $K_{2,1}$ is bounded and compact from $L_{3}$ to $L_{2}$. Analogously by Theorem 3.2 with $\phi_{2}(x)=2 x$ we can prove the boundedness and compactness of $K_{2,2}$. Therefore, if $h(z)=\exp (-z)$, then the initial operator $K_{2}$ is bounded and compact from $L_{3}$ to $L_{2}$.

The last example is about a criterion case from Theorem 5.2.

Example 6.3. Let $q=3 / 2, p=3, v=w=1, k(x, y)=\frac{1}{(x y+1)^{4 / 3}}$, $a(x)=x / 2, b(x)=2 x:$

$$
K_{3} f(x)=\int_{x / 2}^{2 x} \frac{f(y) d y}{(x y+1)^{4 / 3}} .
$$

It holds that $k(x, y) \in \mathcal{O}_{a} \cap \mathcal{O}_{b}$ because for $x / 2 \leq z / 2=a(z) \leq y \leq$ $b(x)=2 x \leq 2 z$ we have $k(x, a(z))=\frac{1}{x \cdot z / 2+1} \leq \frac{1}{x \cdot y / 4+1}<4 k(x, y)$, $k(z, y)=\frac{1}{z y+1} \leq k(x, y), k(x, a(z))+k(z, y)>\frac{1}{x \cdot z / 2+1} \geq k(x, y)$ as well as for $z / 2 \leq x / 2=a(x) \leq y \leq b(z)=2 z \leq 2 x$ it holds that 
$k(x, b(z))=\frac{1}{2 x z+1} \leq k(x, y), k(z, y)=\frac{1}{z y+1} \leq \frac{1}{y \cdot x / 4+1}<4 k(x, y)$, $k(x, b(z))+k(z, y)>\frac{1}{z y+1}>k(x, y)$. Since $a^{-1}(y)=2 y$ and $b^{-1}(y)=y / 2$ we write by $(3.2)$

$$
\int_{y / 2}^{\rho(y)} \frac{d x}{(x y+1)^{2}}=\int_{\rho(y)}^{2 y} \frac{d x}{(x y+1)^{2}} \Longrightarrow \int_{y \cdot \rho(y)+1}^{y^{2} / 2+1} d z^{-1}=\int_{2 y^{2}+1}^{y \cdot \rho(y)+1} d z^{-1},
$$

which yields

$$
\rho(y)=\frac{y\left(4 y^{2}+5\right)}{5 y^{2}+4} .
$$

Since $a=b^{-1}$ we have by (3.1) that

$$
\sigma(x)=\frac{x\left(4 x^{2}+5\right)}{5 x^{2}+4}
$$

too. Important that $\rho=\sigma$ are strictly increasing functions. Thus, since $q=p^{\prime}=3 / 2, p=q^{\prime}=3, r=3, a=b^{-1}$ and $\left(4 t^{2}+5\right) /\left(5 t^{2}+4\right) \leq 5 / 4$ we obtain the condition

$$
\begin{array}{r}
B_{\rho=\sigma}^{3}=\frac{27}{2} \int_{0}^{\infty} \frac{t^{3}\left(4 t^{2}+5\right) d t}{\left(t^{2}+2\right)^{2}\left(2 t^{2}+1\right)^{2}\left(5 t^{2}+4\right)} \leq \frac{27 \cdot 5}{2 \cdot 4} \int_{0}^{\infty} \frac{t^{3} d t}{\left(t^{2}+2\right)^{2}\left(2 t^{2}+1\right)^{2}} \\
\quad<\frac{135}{8} \int_{0}^{\infty} \frac{t d t}{\left(t^{2}+2\right)\left(2 t^{2}+1\right)^{2}} \leq \frac{135}{32} \int_{1}^{\infty} \frac{d z}{z^{3}}=\frac{135}{64}<\infty
\end{array}
$$

which is necessary and sufficient for the boundedness and compactness of $K_{3}$ by Theorem 5.2 (b). This fact can be also proved by using Theorem 5.1 .

Acknowledgment. The author thanks Professor Vladimir Stepanov and Professor Sten Kaijser for helpful discussions, suggestions and valuable remarks. The work is supported by the Swedish Institute (Project 00105/2007 Visby Programme 382). The work is also partially supported by the Russian Foundation for Basic Research (Projects 07-01-00054, 09-0198516) and by the Far-Eastern Branch of the Russian Academy of Sciences (Project 09-II-CO-01-003).

\section{References}

[1] A. L. Bernardis, F. J. Martin-Reyes and P. Ortega Salvador, Weighted inequalities for Hardy-Steklov operators, Canad. J. Math., 59 (2007), 276-295. 
[2] A. L. Bernardis, F. J. Martin-Reyes and P. Ortega Salvador, Weighted weak type inequalities for generalized Hardy operators, J. Inequal. Appl., 2006, Art. ID 62426, 10 pp.

[3] T. Chen and G. Sinnamon, Generalized Hardy operators and normalizing measures, J. Ineq. Appl., 7 (2002), 829-866.

[4] A. Gogatishvili and J. Lang, The generalized Hardy operators with kernel and variable integral limits in Banach function spaces, J. Inequal. Appl., 4 (1999), 1-16.

[5] M. L. Goldman, H. P. Heinig, and V. D. Stepanov, On the principle of duality in Lorentz spaces, Canad. J Math., 48 (1996), 959-979.

[6] H. P. Heinig and G. Sinnamon, Mapping properties of integral averaging operators, Studia Math., 129 (1998), 157-177.

[7] E. Lomakina and V. D. Stepanov, On the Hardy-type integral operators in Banach function spaces, Publ. Mat., 42 (1998), 165-194.

[8] V. D. Stepanov, Weighted norm inequalities of Hardy type for the class of integral operators, J. London Math. Soc., 50 (1994), 105-120.

[9] V. D. Stepanov and E. P. Ushakova, On integral operators with variable limits of integration, Proc. Steklov Inst. Math., 232 (2001), 290-309.

[10] V. D. Stepanov and E. P. Ushakova, Hardy operator with variable limits on monotone functions, J. Funct. Spaces Appl., 1 (2003), 1-15.

[11] V. D. Stepanov and E. P. Ushakova, On the geometric mean operator with variable limits of integration, Proc. Steklov Inst. Math., 260 (2008), 254-278.

Computing Centre of Far Eastern Branch of Russian Academy of Sciences 680000 Khabarovsk, Russia

RUSSIA

(E-mails : elenau@inbox.ru and eu511@york.ac.uk)

(Received : January 2009) 


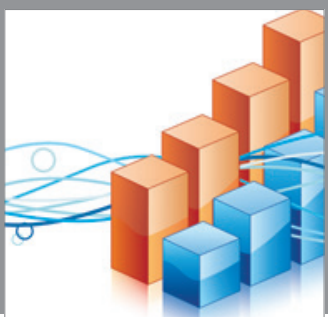

Advances in

Operations Research

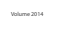

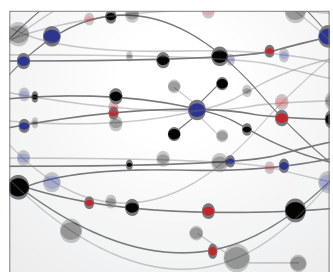

\section{The Scientific} World Journal
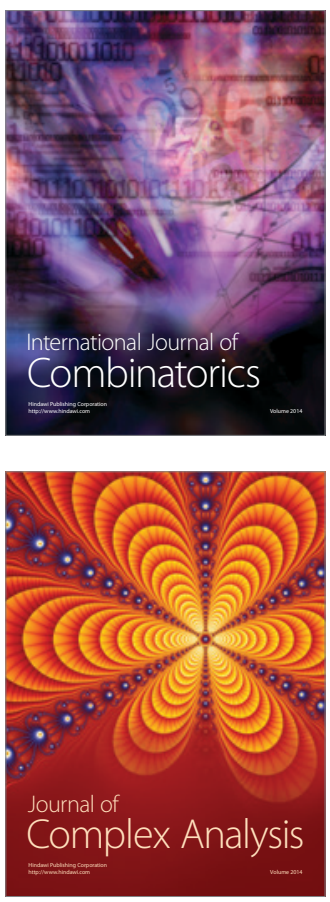

International Journal of

Mathematics and

Mathematical

Sciences
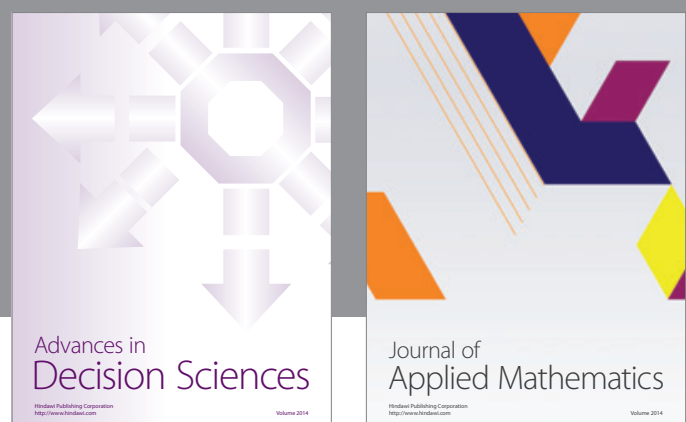

Journal of

Applied Mathematics
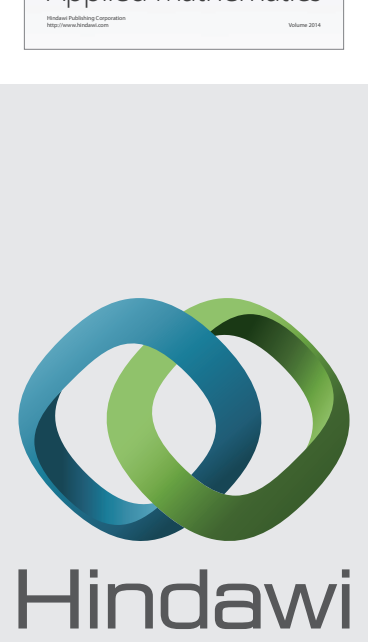

Submit your manuscripts at http://www.hindawi.com
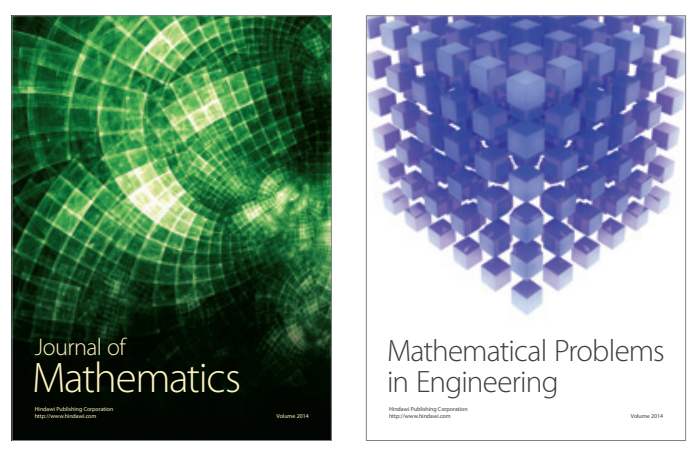

Mathematical Problems in Engineering
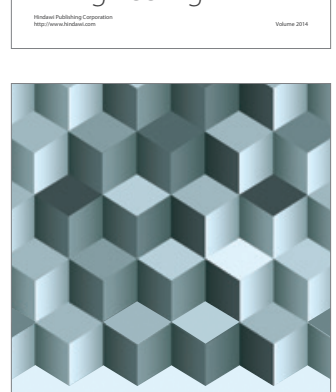

Journal of

Function Spaces
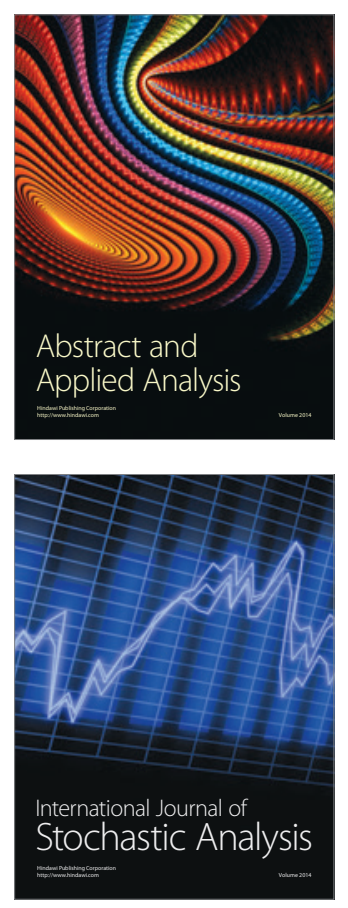

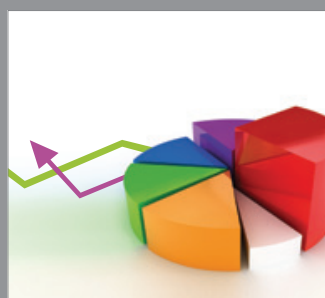

ournal of

Probability and Statistics

Promensencen
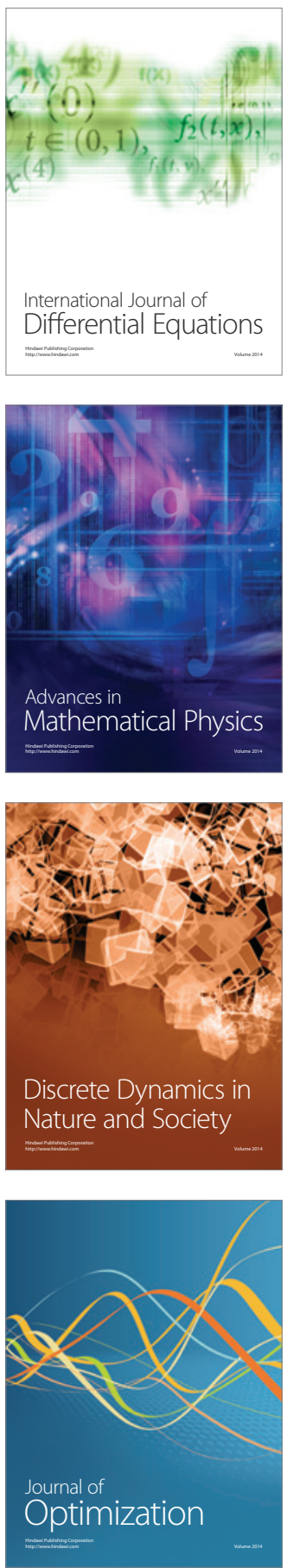